\title{
Organocatalytic Asymmetric Synthesis of Functionalized 1,3,5-Triarylpyrroli- din-2-ones via an Aza-Michael/Aldol Domino Reaction
}

\author{
Céline Joie, Kristina Deckers, Dieter Enders* \\ Institute of Organic Chemistry, RWTH Aachen University, Landoltweg 1, 52074 Aachen, Germany \\ Fax +49(241)8092127; E-mail: enders@rwth-aachen.de
}

Received: 10.12.2013; Accepted: 13.12.2013

License terms: (c)

\begin{abstract}
The organocatalytic asymmetric synthesis of functionalized 1,3,5-triarylpyrrolidin-2-ones bearing three contiguous stereocenters through an aza-Michael/aldol domino reaction of $\alpha$ ketoamides with $\alpha, \beta$-unsaturated aldehydes is described. The domino products were further derivatized by aldehyde olefination under one-pot conditions. The reaction proceeds with excellent diastereoselectivities (>20:1) and good to excellent enantioselectivities (60-96\% ee).
\end{abstract}

Key words: organocatalysis, domino reactions, asymmetric synthesis, pyrrolidin-2-ones, aza-Michael addition

The polysubstituted pyrrolidin-2-one or $\gamma$-lactam core is a crucial structural feature of numerous pharmaceuticals and natural products of diverse complexity possessing various biological activities (Figure 1). Prominent examples are lactacystin $(\mathbf{A})^{1}$ isolated from Streptomyces and acting against multiple cancer cell lines as a proteasome inhibitor; clausenamide (B), ${ }^{2}$ rolipram $(\mathbf{C}),{ }^{3}$ known as an anti-inflammatory and antidepressant agent; and cotinine (D) ${ }^{4}$ a metabolite of nicotine also used as an antidepressant. Even closer to the title compounds, 3,5-diarylpyrrolidin-2-ones $\mathbf{E}$ have shown activity as neurokinin-1 (NK1) antagonists $^{5}$ or as endothelin receptor antagonists. ${ }^{6}$ As a result, multiple synthetic pathways have been developed in order to access these nitrogen-containing five-membered heterocycles. ${ }^{7}$ Various diastereoselective syntheses of these polysubstituted pyrrolidin-2-ones have already been reported, ${ }^{8}$ whereas enantioselective methods are less developed. ${ }^{9}$

The rapidly growing field of asymmetric organocatalysis has proved to be a powerful tool for the synthesis of chiral molecules and it has been widely applied in synthesis. ${ }^{10}$ Particularly, organocatalyzed domino reactions have shown their efficiency when it comes to the one-pot formation of several bonds yielding highly substituted compounds with excellent diastereo- and enantioselectivities. ${ }^{11}$ Secondary amine catalysts working via iminium or enamine activation of carbonyl compounds have been efficiently used in simple, ${ }^{12}$ triple, ${ }^{13}$ and quadruple ${ }^{14}$ cascade reactions.

The organocatalyzed aza-Michael addition has been frequently used in the asymmetric synthesis of nitrogen-

SYNTHESIS 2014, 46, 0799-0808

Advanced online publication: 09.01.2014

DOI: 10.1055/s-0033-1340565; Art ID: SS-2013-Z0800-OP

(C) Georg Thieme Verlag Stuttgart · New York containing molecules. ${ }^{15}$ Domino reactions with substrates that bear a nucleophilic nitrogen atom and electrophilic centers have been used to achieve the synthesis of heterocycles. $\alpha$-Ketoamides have already been employed in organocatalysis, ${ }^{16}$ although the nucleophilicity of their nitrogen atom has not been exploited yet in an azaMichael addition. Furthermore, these molecules also possess two electrophilic centers that make them ideal partners for the design of new cascade reactions.

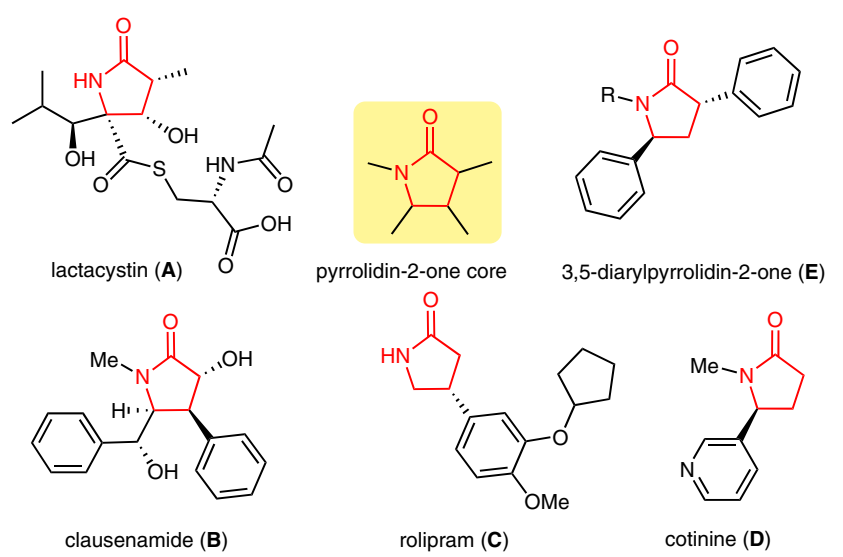

Figure 1 Typical examples of pyrrolidin-2-one-containing natural products and pharmaceuticals

Herein we wish to report an organocatalyzed asymmetric synthesis of densely substituted pyrrolidin-2-ones via an aza-Michael/aldol domino reaction sequence of $\alpha$-ketoamides with $\alpha, \beta$-unsaturated aldehydes, followed by aldehyde olefination in a one-pot fashion (Scheme 1).

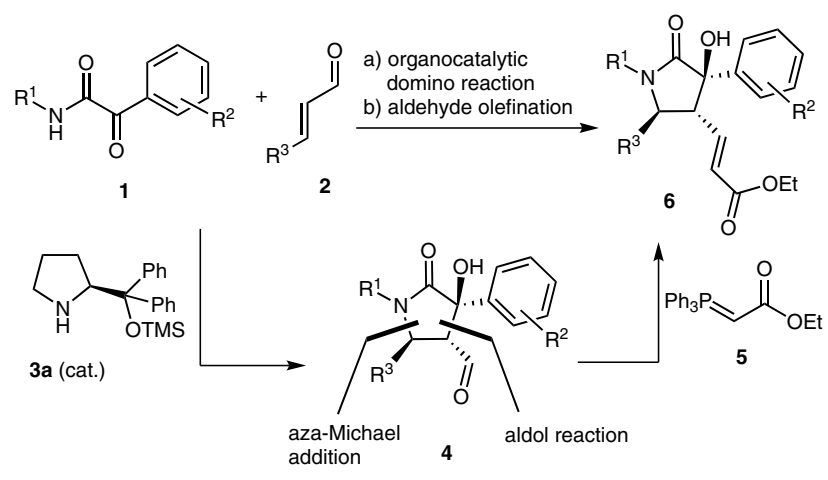

Scheme 1 Asymmetric one-pot synthesis of functionalized 1,3,5-triarylpyrrolidin-2-one derivatives by an organocatalytic simple domino reaction followed by Wittig olefination 
We began by investigating the reaction of 2-oxo- $N, 2$-diphenylacetamide (1a), cinnamaldehyde (2a), and 20 mol\% of the diphenylprolinol trimethylsilyl ether catalyst (S)-3a (Table 1). Although chloroform, dichloromethane, and toluene yielded mainly the condensation product 7 and its isomerized equivalent 8 (entries 1-3), ethanol, methanol, and dimethyl sulfoxide appeared to furnish the desired product 4 . We realized that the domino product itself was not suitable for purification and analysis due to its relative instability. As a consequence, the aldehyde 4 was trapped in a one-pot fashion with 1.5 equivalents of the stabilized Wittig reagent 5 converting it into the more stable $\alpha, \beta$-unsaturated ester $\mathbf{6}$.

Table 1 Optimization of the Reaction Conditions ${ }^{\mathrm{a}}$<smiles>O=C(Nc1ccccc1)C(=O)c1ccccc1</smiles>

1a

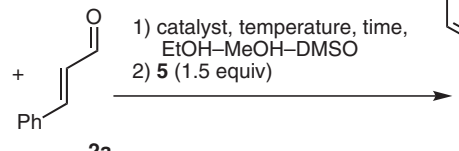

2a

$3 \mathrm{a}(20 \mathrm{~mol} \%)$, r.t. $\mathrm{CH}_{2} \mathrm{Cl}_{2}, \mathrm{CHCl}_{3}$ or toluene
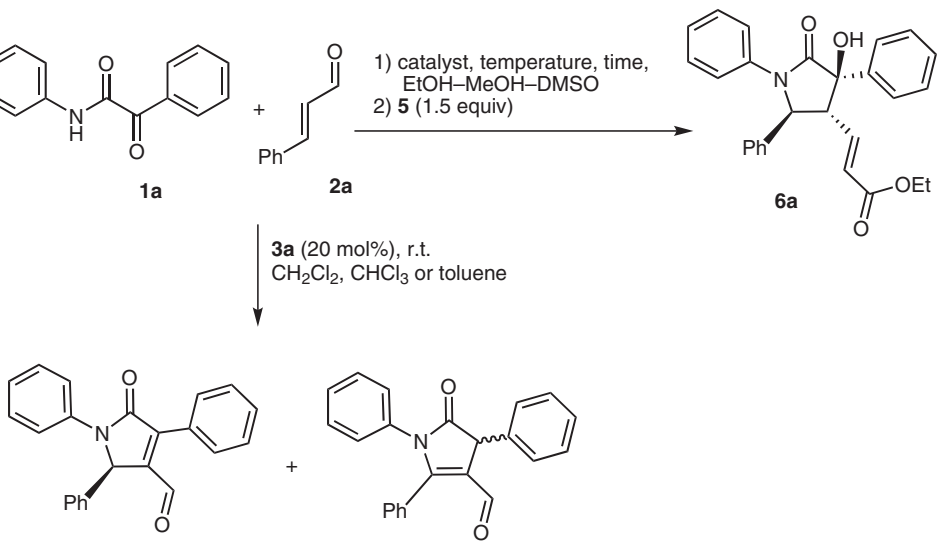

\begin{tabular}{|c|c|c|c|c|c|}
\hline 3 & 3b & & & & \\
\hline Entry & Catalyst & Solvent & Time $^{\mathrm{b}}(\mathrm{h})$ & Ratio 1a/2a & Yield $^{\mathrm{c}}(\%)$ of $\mathbf{6 a}$ \\
\hline 1 & $3 a$ & $\mathrm{CH}_{2} \mathrm{Cl}_{2}$ & 4.5 & $1: 1$ & $-{ }^{\mathrm{d}}$ \\
\hline 2 & $3 a$ & $\mathrm{CHCl}_{3}$ & 4.5 & $1: 1$ & $-{ }^{\mathrm{d}}$ \\
\hline 3 & $3 a$ & toluene & 4.5 & $1: 1$ & $-^{\mathrm{d}}$ \\
\hline 4 & $3 a$ & DMSO & 4.5 & $1: 1$ & 10 \\
\hline 5 & $3 a$ & $\mathrm{MeOH}$ & 4.5 & $1: 1$ & 51 \\
\hline 6 & $3 a$ & $\mathrm{EtOH}$ & 4.5 & $1: 1$ & 50 \\
\hline 7 & $3 b$ & $\mathrm{EtOH}$ & 4.5 & $1: 1$ & 10 \\
\hline 8 & $3 c$ & $\mathrm{EtOH}$ & 4.5 & $1: 1$ & - \\
\hline 9 & 3d & $\mathrm{EtOH}$ & 4.5 & $1: 1$ & - \\
\hline 10 & $3 a$ & $\mathrm{EtOH}$ & 3 & $1: 1$ & 42 \\
\hline 11 & $3 a$ & $\mathrm{EtOH}$ & 8 & $1: 1$ & 46 \\
\hline 12 & $3 a$ & $\mathrm{EtOH}$ & 16 & $1: 1$ & $-^{\mathrm{d}}$ \\
\hline 13 & $3 a$ & $\mathrm{EtOH}$ & 4.5 & $1: 1.5$ & - \\
\hline 14 & $3 a$ & $\mathrm{EtOH}$ & 4.5 & $1.5: 1$ & 65 \\
\hline 15 & $3 a$ & $\mathrm{EtOH}$ & 4.5 & $2: 1$ & 66 \\
\hline
\end{tabular}

${ }^{a}$ Unless otherwise noted, all reactions were performed on a $0.33-\mathrm{mmol}$ scale using the indicated ratio of $\mathbf{1 a} / \mathbf{2 a}$, catalyst (20 mol\%), solvent (1.5 $\mathrm{mL}$ ), r.t., for the indicated time, followed by addition of the Wittig reagent (1.5 equiv) with reaction overnight.

${ }^{\mathrm{b}}$ Time for the domino reaction before the introduction of the Wittig reagent.

${ }^{\mathrm{c}}$ Isolated yield.

${ }^{\mathrm{d}}$ A mixture of $\mathbf{7}$ and $\mathbf{8}$ was obtained. 
After performing a short catalyst screening and an evaluation of the optimum reaction conditions, we concluded that the best results were obtained using 1.5 equivalents of the $\alpha$-ketoamide 1a, 1 equivalent of the cinnamaldehyde $\mathbf{2 a}$ and $20 \mathrm{~mol} \%$ of the catalyst 3a. The reaction was performed in $1.5 \mathrm{~mL}$ of ethanol for 4.5 hours at room temperature before the addition of 1.5 equivalents of the Wittig reagent (entry 15). A decrease in the catalyst loading gave lower yields, while increasing it gave no significant improvement; using a longer reaction time before the addition of the Wittig reagent also did not improve the outcome of the reaction (entry 11). Performing the reaction overnight yielded almost exclusively dehydrated products $\mathbf{7}$ and $\mathbf{8}$ (entry 12).

With the optimized conditions in hand, we explored the scope of the reaction. Initially we varied the $\alpha, \beta$-unsaturated aldehyde moiety $\mathbf{2}$ (Table 2 ). Although the yields show high variations (between 20 and $73 \%$ ), the reaction tolerates electron-withdrawing as well as electron-donating groups giving products $\mathbf{6 a}-\mathbf{i}$ with good to excellent en- antioselectivities (entries 1-9). Heteroaryl substituents are also well accepted giving $\mathbf{6 h}, \mathbf{i}$, although a lower asymmetric induction was observed with the furyl derivative 6h (entries 8 and 9). Aliphatic residues did not lead to any satisfactory results.

The extension of the scope regarding the $\alpha$-ketoamide substrate was also investigated. For this purpose we synthesized derivatives bearing different groups on both aromatic rings $\left(\mathrm{R}^{1}, \mathrm{R}^{2}\right)$, and performed the cascade reactions using the optimum conditions (entries 10-13). The yields of $\mathbf{6 j - m}$ as well as the enantioselectivities were in accordance with the results obtained previously. Other $\alpha$-ketoamide derivatives bearing nonaromatic residues were also tested in the cascade reaction, however these did not react in the desired fashion, with the exception of the 2-oxo-2phenyl- $N$-tosylacetamide (1f), which gave the desired pyrrolidin-2-one 6n in very good yield with excellent enantioselectivity (entry 14).

In the present transformation we assume that the reaction is initiated by an aza-Michael addition of the nucleophilic

Table 2 Reaction of $\alpha$-Ketoamides 1 with $\alpha, \beta$-Unsaturated Aldehydes $\mathbf{2}^{\mathrm{a}}$

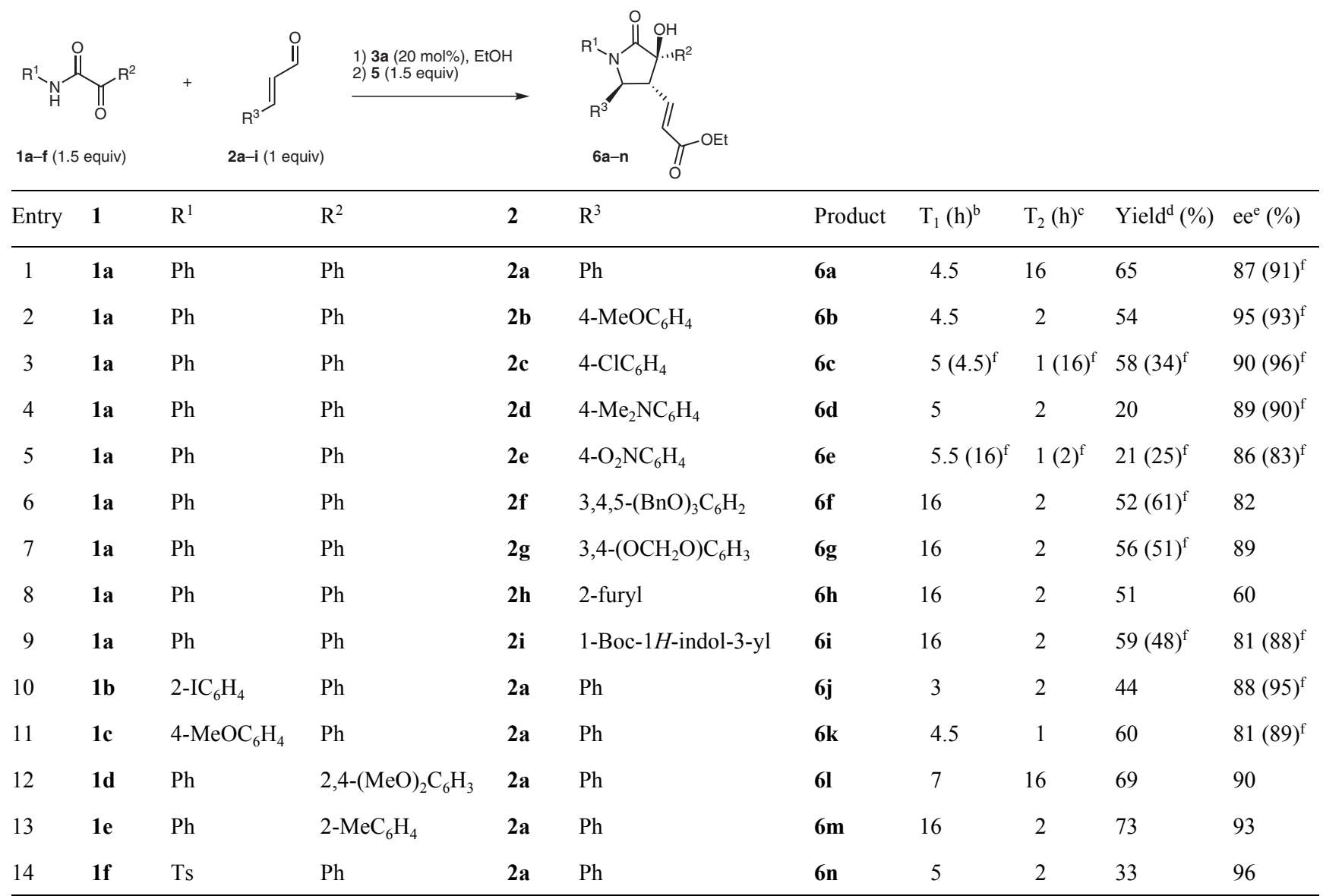

${ }^{a}$ All the reactions were run on a 0.33 -mmol scale with $\alpha$-ketoamide (1.5 equiv), $\alpha, \beta$-unsaturated aldehyde ( 1 equiv), 3a (20 mol\%), EtOH (1.5 $\mathrm{mL}$ ), r.t. All the products were obtained as a single diastereomer.

${ }^{\mathrm{b}}$ Time for the domino reaction before the addition of the Wittig reagent 5 (1.5 equiv).

${ }^{\mathrm{c}}$ Time for the olefination.

${ }^{\mathrm{d}}$ Yield of the isolated product $\mathbf{6 a}-\mathbf{n}$ after flash column chromatography.

${ }^{\mathrm{e}}$ Determined by HPLC on a chiral stationary phase.

${ }^{\mathrm{f}}$ Values in parentheses correspond to the results obtained with the catalyst $(R)-\mathbf{3 a}$. 


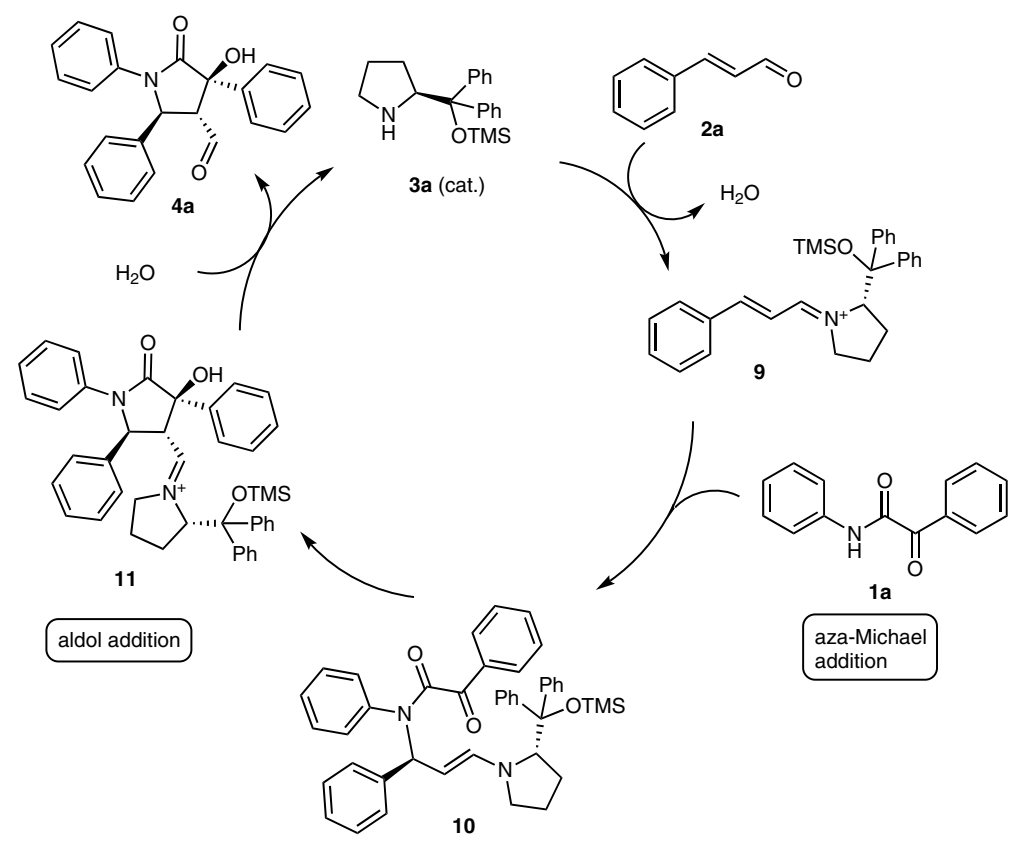

Scheme 2 Proposed mechanism for the domino reaction

nitrogen of the $\alpha$-ketoamide to the iminium-activated $\alpha, \beta$ unsaturated aldehyde 9 . This yields the acyclic intermediate $\mathbf{1 0}$ that undergoes an aldol addition cyclization due to the enamine activation providing the intermediate 11, and the product $\mathbf{4 a}$ after hydrolysis and return of the catalyst (Scheme 2). The expected transition states for the iminium [Scheme 3 (a)] as well as for the enamine activation [Scheme 3 (b)] allowed us to predict the relative and absolute configuration of the products. They were confirmed by NOESY experiments on $\mathbf{6 1}$ [Scheme 3 (c)].
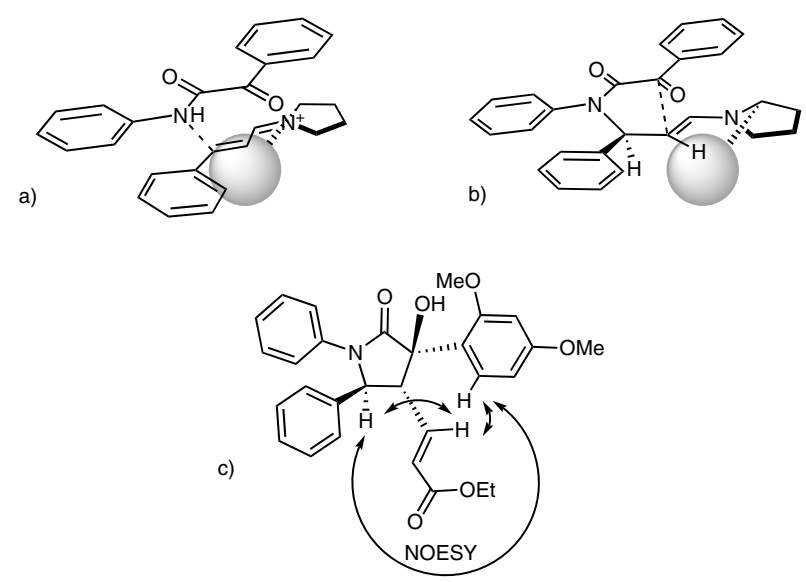

Scheme 3 Proposed transition states for (a) iminium activation, (b) enamine activation, (c) determination of the relative configuration by NOESY measurements

In conclusion, we have developed a new convenient organocatalytic method for the asymmetric synthesis of functionalized 1,3,5-triarylpyrrolidin-2-ones in good yields, virtually complete diastereoselectivities, and very good enantioselectivities via an aza-Michael/aldol addi- tion domino reaction between $\alpha$-ketoamides and $\alpha, \beta$-unsaturated aldehydes. As the pyrrolidin-2-one substructure is widely present in a large number of biologically active molecules, our protocol could provide a new approach for these $\gamma$-lactams. The applications of the method described here are currently being investigated.

Unless otherwise noted, all commercially available compounds were used without further purification. Preparative column chromatography SIL G-25 UV252 from Macherey \& Nagel, particle size $0.040-0.063 \mathrm{~nm}(230-240 \mathrm{mesh}$, flash). Visualization of the developed TLC plates was performed with UV irradiation $(254 \mathrm{~nm})$ and by staining with vanillin stain. Optical rotations were measured on a Perkin-Elmer 241 polarimeter. Mass spectra were recorded on a Finnigan SSQ7000 (EI $70 \mathrm{eV}$ ) spectrometer and HRMS on a Thermo Fisher Scientific Orbitrap XL spectrometer. IR spectra were recorded on a Perkin-Elmer FT-IR Spectrum 100 using ATR-Unit. ${ }^{1} \mathrm{H}$ and ${ }^{13} \mathrm{C}$ spectra were recorded at r.t. on Varian Mercury 600 or Inova 400 instruments with TMS as an internal standard. Analytical HPLC was performed on a Hewlett-Packard 1100 Series instrument using chiral stationary phases (Daicel AD, Daicel AS, Daicel IA, Daicel OD, Diacel OJ, or Chiralpak IC). $\alpha$-Ketoamide 1d was prepared from ethyl 2-(2,4-dimethoxyphenyl)-2-oxoacetate via 2-(2,4dimethoxyphenyl)-2-oxoacetic acid; $\alpha$-ketoamide 1e was prepared from $o$-methylmandelic acid via 2-methylphenylglyoxylic acid.

\section{a-Ketoamides 1; General Procedure}

To a solution of phenylglyoxylic acid ( $1.774 \mathrm{~g}, 12 \mathrm{mmol}, 1$ equiv) in $N, N$-dimethylacetamide $(30 \mathrm{~mL})$ cooled to $-15^{\circ} \mathrm{C}, \mathrm{SOCl}_{2}(1.0$ $\mathrm{mL}, 13.8 \mathrm{mmol}, 1.15$ equiv) was added dropwise. After stirring for $1 \mathrm{~h}$ at this temperature, aniline $(1.5 \mathrm{~mL}, 16.7 \mathrm{mmol}, 1.4$ equiv) was added and the mixture was stirred for $3 \mathrm{~h}$ at temperatures between $-10{ }^{\circ} \mathrm{C}$ and $0{ }^{\circ} \mathrm{C}$, then poured onto a mixture of ice and $\mathrm{H}_{2} \mathrm{O}$ and stirred at r.t. overnight. The solid formed was dissolved in $\mathrm{Et}_{2} \mathrm{O}$ (50 $\mathrm{mL})$ and extracted with $\mathrm{Et}_{2} \mathrm{O}(3 \times 20 \mathrm{~mL})$. The combined organic layers were washed with $\mathrm{H}_{2} \mathrm{O}$ and brine, dried $\left(\mathrm{Na}_{2} \mathrm{SO}_{4}\right)$, and concentrated in vacuo before purification by flash column chromatography. 


\section{Domino Reaction; General Procedure}

A solution of $\alpha$-ketoamide 1 ( $0.5 \mathrm{mmol}, 1.5$ equiv), $\alpha, \beta$-unsaturated aldehyde 2 ( $0.33 \mathrm{mmol}, 1$ equiv), and (S)-TMS-diphenylprolinol catalyst 3a (23 mg, $0.066 \mathrm{mmol}, 0.2$ equiv) in EtOAc $(1.5 \mathrm{~mL})$ was stirred at r.t. After the indicated period, the Wittig reagent $5(0.174$ $\mathrm{g}, 0.5 \mathrm{mmol}, 1.5$ equiv) was added and the reaction was stirred at r.t. for the indicated time. Brine $(20 \mathrm{~mL})$ was added and the product was extracted with $\mathrm{Et}_{2} \mathrm{O}(3 \times 15 \mathrm{~mL})$. The combined organic layers were dried $\left(\mathrm{MgSO}_{4}\right)$, concentrated in vacuo, and purified by flash column chromatography ( $n$-pentane-EtOAc, $3: 1$ ).

\section{2-Ox0- $N$,2-diphenylacetamide (1a)}

Following the general procedure using aniline $(1.5 \mathrm{~mL}, 16.7 \mathrm{mmol}$ 1.4 equiv) and phenylglyoxylic acid ( $1.774 \mathrm{~g}, 12 \mathrm{mmol}, 1$ equiv) with purification of the crude product by flash column chromatography ( $n$-pentane- $\left.\mathrm{Et}_{2} \mathrm{O}, 4: 1\right)$ gave $\mathbf{1 a}(2.69 \mathrm{~g}, 99 \%)$ as a yellow solid; $R_{f}=0.5$ (n-pentane- $\left.\mathrm{Et}_{2} \mathrm{O}, 4: 1\right)$

${ }^{1} \mathrm{H} \mathrm{NMR}\left(600 \mathrm{MHz}, \mathrm{CDCl}_{3}\right): \delta=7.18\left(\mathrm{~m}, 1 \mathrm{H}, \mathrm{CH}_{\mathrm{Ar}}\right), 7.38(\mathrm{~m}, 2 \mathrm{H}$, $\left.\mathrm{CH}_{\mathrm{Ar}}\right), 7.49\left(\mathrm{~m}, 2 \mathrm{H}, \mathrm{CH}_{\mathrm{Ar}}\right), 7.64\left(\mathrm{~m}, 1 \mathrm{H}, \mathrm{CH}_{\mathrm{Ar}}\right), 7.69(\mathrm{dd}, J=1.2$, $\left.8.4 \mathrm{~Hz}, 2 \mathrm{H}, \mathrm{CH}_{\mathrm{Ar}}\right), 8.40\left(\mathrm{dd}, J=1.2,8.4 \mathrm{~Hz}, 2 \mathrm{H}, \mathrm{CH}_{\mathrm{Ar}}\right.$ ), 8.97 (br s, $1 \mathrm{H}, \mathrm{NH})$

${ }^{13} \mathrm{C}$ NMR $\left(150 \mathrm{MHz}, \mathrm{CDCl}_{3}\right): \delta=119.9\left(\mathrm{CH}_{\mathrm{Ar}}\right), 125.3\left(\mathrm{CH}_{\mathrm{Ar}}\right)$, $128.5\left(\mathrm{CH}_{\mathrm{Ar}}\right), 129.2\left(\mathrm{CH}_{\mathrm{Ar}}\right), 131.4\left(\mathrm{CH}_{\mathrm{Ar}}\right), 133.0(\mathrm{C}), 134.6\left(\mathrm{CH}_{\mathrm{Ar}}\right)$, $136.6(\mathrm{C}), 158.9$ (NCO), $187.4(\mathrm{CO})$.

\section{$N$-(2-Iodophenyl)-2-oxo-2-phenylacetamide (1b)}

Following the general procedure using 2-iodoaniline $(1.41 \mathrm{~g}, 6.4$ mmol, 1.4 equiv) and phenylglyoxylic acid $(0.69 \mathrm{~g}, 12 \mathrm{mmol}, 1$ equiv) with purification of the crude product by flash column chromatography ( $n$-pentane- $\left.\mathrm{Et}_{2} \mathrm{O} 6: 1\right)$ gave $1 \mathrm{~b}(0.88 \mathrm{~g}, 54 \%)$ as a yellow solid; mp $47{ }^{\circ} \mathrm{C} ; R_{f}=0.43$ (n-pentane- $\mathrm{Et}_{2} \mathrm{O}, 9: 1$ ).

IR (ATR): 3612, 3299, 3055, 1697, 1661, 1577, 1514, 1433, 1271, $1155,986,943,870,801,740,676 \mathrm{~cm}^{-1}$.

${ }^{1} \mathrm{H}$ NMR $\left(600 \mathrm{MHz}, \mathrm{CDCl}_{3}\right): \delta=6.93\left(\mathrm{t}, J=7.2 \mathrm{~Hz}, 1 \mathrm{H}, \mathrm{CH}_{\mathrm{Ar}}\right)$, $7.42\left(\mathrm{t}, J=7.2 \mathrm{~Hz}, 1 \mathrm{H}, \mathrm{CH}_{\mathrm{Ar}}\right), 7.53\left(\mathrm{t}, J=7.2 \mathrm{~Hz}, 2 \mathrm{H}, \mathrm{CH}_{\mathrm{Ar}}\right), 7.67$ $\left(\mathrm{t}, J=7.2 \mathrm{~Hz}, 1 \mathrm{H}, \mathrm{CH}_{\mathrm{Ar}}\right), 7.85\left(\mathrm{~d}, J=7.8 \mathrm{~Hz}, 1 \mathrm{H}, \mathrm{CH}_{\mathrm{Ar}}\right), 8.41(\mathrm{~d}$ $\left.J=7.8 \mathrm{~Hz}, 1 \mathrm{H}, \mathrm{CH}_{\mathrm{Ar}}\right), 8.44\left(\mathrm{~d}, J=7.2 \mathrm{~Hz}, 2 \mathrm{H}, \mathrm{CH}_{\mathrm{Ar}}\right), 9.44$ (br s, $1 \mathrm{H}, \mathrm{NH})$.

${ }^{13} \mathrm{C}$ NMR $\left(150 \mathrm{MHz}, \mathrm{CDCl}_{3}\right): \delta=90.5(\mathrm{CI}), 121.7\left(\mathrm{CH}_{\mathrm{Ar}}\right), 127.1$ $\left(\mathrm{CH}_{\mathrm{Ar}}\right), 128.9\left(\mathrm{CH}_{\mathrm{Ar}}\right), 129.6\left(\mathrm{CH}_{\mathrm{Ar}}\right), 131.8\left(\mathrm{CH}_{\mathrm{Ar}}\right), 133.3(\mathrm{C}), 135.1$ $\left(\mathrm{CH}_{\mathrm{Ar}}\right), 137.6(\mathrm{C}), 139.6\left(\mathrm{CH}_{\mathrm{Ar}}\right), 152.2(\mathrm{NCO}), 187.0(\mathrm{CO})$.

MS (EI, $70 \mathrm{eV}): m / z(\%)=351$ (33), 244 (12), 224 (100), 196 (13), 119 (12), 77 (65), 76 (18), 55 (12), 54 (15)

Anal. Calcd for $\mathrm{C}_{14} \mathrm{H}_{10} \mathrm{INO}_{2}$ : C, 47.89; H, 2.87; N, 3.99. Found: C, $47.60 ; \mathrm{H}, 2.60 ; \mathrm{N}, 3.89$.

\section{$\mathrm{N}$-(4-Methoxyphenyl)-2-oxo-2-phenylacetamide (1c)}

Following the general procedure using $p$-anisidine $(431 \mathrm{mg}, 3.5$ mmol, 1.4 equiv) and phenylglyoxylic acid (376 mg, $2.5 \mathrm{mmol}, 1$ equiv) with purification of the crude product by flash column chromatography ( $n$-pentane- $\left.\mathrm{Et}_{2} \mathrm{O}, 2: 1\right)$ gave $1 \mathrm{c}(495 \mathrm{mg}, 76 \%)$ as a yellow solid; $\mathrm{mp} 103{ }^{\circ} \mathrm{C} ; R_{f}=0.43$ (n-pentane- $\left.\mathrm{Et}_{2} \mathrm{O}, 2: 1\right)$.

${ }^{1} \mathrm{H} \mathrm{NMR}\left(600 \mathrm{MHz}, \mathrm{CDCl}_{3}\right): \delta=3.82\left(\mathrm{~s}, 3 \mathrm{H}, \mathrm{CH}_{3}\right), 6.92(\mathrm{~d}, J=8.4$ $\left.\mathrm{Hz}, 2 \mathrm{H}, \mathrm{CH}_{\mathrm{Ar}}\right), 7.50\left(\mathrm{t}, J=7.8 \mathrm{~Hz}, 2 \mathrm{H}, \mathrm{CH}_{\mathrm{Ar}}\right), 7.64\left(\mathrm{~m}, 3 \mathrm{H}, \mathrm{CH}_{\mathrm{Ar}}\right)$, $8.41\left(\mathrm{~d}, J=7.8 \mathrm{~Hz}, 2 \mathrm{H}, \mathrm{CH}_{\mathrm{Ar}}\right), 8.89(\mathrm{br} \mathrm{s}, 1 \mathrm{H}, \mathrm{NH})$.

${ }^{13} \mathrm{C} \mathrm{NMR}\left(150 \mathrm{MHz}, \mathrm{CDCl}_{3}\right): \delta=55.5\left(\mathrm{OCH}_{3}\right), 114.4\left(\mathrm{CH}_{\mathrm{Ar}}\right)$, $121.5\left(\mathrm{CH}_{\mathrm{Ar}}\right), 128.5\left(\mathrm{CH}_{\mathrm{Ar}}\right), 129.8(\mathrm{C}), 131.4\left(\mathrm{CH}_{\mathrm{Ar}}\right), 133.2(\mathrm{C})$, $134.5\left(\mathrm{CH}_{\mathrm{Ar}}\right), 157.1\left(\mathrm{COCH}_{3}\right), 158.7(\mathrm{NCO}), 187.6(\mathrm{CO})$

\section{Ethyl 2-(2,4-Dimethoxyphenyl)-2-oxoacetate}

To a solution of 1,3-dimethoxybenzene $(1.31 \mathrm{~mL}, 10 \mathrm{mmol}, 1$ equiv) in anhyd $\mathrm{CH}_{2} \mathrm{Cl}_{2}(20 \mathrm{~mL})$ cooled to $0{ }^{\circ} \mathrm{C}$ under argon $\mathrm{SnCl}_{4}$ was carefully added (1.4 mL, $12 \mathrm{mmol}, 1.2$ equiv), and the solution was stirred for $30 \mathrm{~min}$ at r.t. After cooling to $0{ }^{\circ} \mathrm{C}$, ethyl oxalyl chloride $(3.36 \mathrm{~mL}, 30 \mathrm{mmol}, 3$ equiv) was added dropwise, followed by $\mathrm{MeNO}_{2}(15 \mathrm{~mL})$. The resulting dark purple solution was stirred for
$1 \mathrm{~h}$ at r.t. The reaction was quenched by the careful addition of sat. aq $\mathrm{NaHCO}_{3}(10 \mathrm{~mL})$, the $\mathrm{pH}$ was brought to 10 by the addition of $25 \% \mathrm{NaOH}(10 \mathrm{~mL})$ and the mixture was stirred at r.t. for $1 \mathrm{~h}$. The crude product was extracted with EtOAc $(3 \times 25 \mathrm{~mL})$ and the combined organic layers were washed with $\mathrm{H}_{2} \mathrm{O}$, dried $\left(\mathrm{MgSO}_{4}\right)$, and evaporated in vacuo. Flash column chromatography ( $n$-pentane$\left.\mathrm{Et}_{2} \mathrm{O} 1: 1\right)$ of the residue gave the product $(1.133 \mathrm{~g}, 47.5 \%)$ as a colorless oil; $R_{f}=0.26$ ( $n$-pentane- $\left.\mathrm{Et}_{2} \mathrm{O}, 1: 1\right)$.

IR (ATR): 3098, 2980, 2846, 1739, 1661, 1594, 1464, 1374, 1302, $1208,1117,1024,919,836,754,673,583,515 \mathrm{~cm}^{-1}$.

${ }^{1} \mathrm{H}$ NMR $\left(600 \mathrm{MHz}, \mathrm{CDCl}_{3}\right): \delta=1.39\left(\mathrm{t}, J=7.2 \mathrm{~Hz}, 3 \mathrm{H}, \mathrm{CH}_{2} \mathrm{CH}_{3}\right)$, $3.85\left(\mathrm{~s}, 3 \mathrm{H}, \mathrm{OCH}_{3}\right), 3.88\left(\mathrm{~s}, 3 \mathrm{H}, \mathrm{OCH}_{3}\right), 4.37(\mathrm{q}, J=7.2 \mathrm{~Hz}, 2 \mathrm{H}$, $\left.\mathrm{CH}_{2}\right), 6.43\left(\mathrm{~d}, J=1.8 \mathrm{~Hz}, 1 \mathrm{H}, \mathrm{CH}_{\mathrm{Ar}}\right), 6.60(\mathrm{dd}, J=1.8,9 \mathrm{~Hz}, 1 \mathrm{H}$, $\left.\mathrm{CH}_{\mathrm{Ar}}\right), 7.91\left(\mathrm{~d}, J=9 \mathrm{~Hz}, 1 \mathrm{H}, \mathrm{CH}_{\mathrm{Ar}}\right)$.

${ }^{13} \mathrm{C}$ NMR $\left(150 \mathrm{MHz}, \mathrm{CDCl}_{3}\right): \delta=14.2\left(\mathrm{CH}_{3}\right), 55.7\left(\mathrm{OCH}_{3}\right), 56.0$ $\left(\mathrm{CH}_{3}\right), 61.6\left(\mathrm{CH}_{2}\right), 98.2\left(\mathrm{CH}_{\mathrm{Ar}}\right), 106.7\left(\mathrm{CH}_{\mathrm{Ar}}\right), 116.0(\mathrm{C}), 132.9$ $\left(\mathrm{CH}_{\mathrm{Ar}}\right), 162.3(\mathrm{C}), 165.9(\mathrm{C}), 166.7(\mathrm{C}), 185.0(\mathrm{C})$.

MS (ESI): $m / z(\%)=261\left(100,[\mathrm{M}+\mathrm{Na}]^{+}\right), 239\left(55,[\mathrm{M}+\mathrm{H}]^{+}\right)$.

Anal. Calcd for $\mathrm{C}_{12} \mathrm{H}_{14} \mathrm{O}_{5}$ : C, 60.50; H, 5.92. Found: C, 60.30; H, 5.79 .

\section{2-(2,4-Dimethoxyphenyl)-2-oxoacetic Acid}

To a solution of ethyl 2-(2,4-dimethoxyphenyl)-2-oxoacetate (1.0 g, $4.2 \mathrm{mmol})$ in $\mathrm{EtOH}(50 \mathrm{~mL})$ was added $2 \mathrm{M}$ aq NaOH $(14 \mathrm{~mL})$. The mixture was stirred for $15 \mathrm{~min}$ at r.t. and the $\mathrm{pH}$ was brought to 1 with $10 \% \mathrm{HCl}$. The product was extracted with $\mathrm{CH}_{2} \mathrm{Cl}_{2}(3 \times 25$ $\mathrm{mL}$ ), dried $\left(\mathrm{MgSO}_{4}\right)$, and the solvent was evaporated. Filtration (silica gel, acetone) gave pure product $(0.69 \mathrm{~g}, 79 \%)$ as a beige solid; $R_{f}=0.4$ (n-pentane-acetone, 1:1).

IR (ATR): 2987, 2945, 2845, 1708, 1649, 1591, 1469, 1422, 1341, $1310,1282,1248,1210,1106,1017,981,898,833,773,736,696$, $671 \mathrm{~cm}^{-1}$.

${ }^{1} \mathrm{H}$ NMR $\left(600 \mathrm{MHz}, \mathrm{CDCl}_{3}\right): \delta=3.90\left(\mathrm{~s}, 6 \mathrm{H}, \mathrm{OCH}_{3}\right), 6.46(\mathrm{~d}$, $\left.J=1.8 \mathrm{~Hz}, 1 \mathrm{H}, \mathrm{CH}_{\mathrm{Ar}}\right), 6.62\left(\mathrm{dd}, J=1.8,8.4 \mathrm{~Hz}, 1 \mathrm{H}, \mathrm{CH}_{\mathrm{Ar}}\right), 7.98$ (d, $J=8.4 \mathrm{~Hz}, 1 \mathrm{H}, \mathrm{CH}_{\mathrm{Ar}}$ ).

${ }^{13} \mathrm{C}$ NMR $\left(150 \mathrm{MHz}, \mathrm{CDCl}_{3}\right): \delta=55.8\left(\mathrm{CH}_{3}\right), 56.1\left(\mathrm{CH}_{3}\right), 98.3$ $\left(\mathrm{CH}_{\mathrm{Ar}}\right), 106.9\left(\mathrm{CH}_{\mathrm{Ar}}\right), 115.3(\mathrm{C}), 133.3\left(\mathrm{CH}_{\mathrm{Ar}}\right), 162.7(\mathrm{C}), 167.1$ (C), $167.1(\mathrm{COOH}), 183.7(\mathrm{CO})$.

Anal. Calcd for $\mathrm{C}_{10} \mathrm{H}_{10} \mathrm{O}_{5}$ : C, 57.14; H, 4.80. Found: C, 57.16; H, 5.09 .

\section{2-(2,4-Dimethoxyphenyl)-2-oxo- $\boldsymbol{N}$-phenylacetamide (1d)}

Following the general procedure using aniline $(0.37 \mathrm{~g}, 4.0 \mathrm{mmol}$, 1.4 equiv) and 2-(2,4-dimethoxyphenyl)-2-oxoacetic acid ( $0.6 \mathrm{~g}$, $2.85 \mathrm{mmol}, 1$ equiv), with purification of the crude product by flash column chromatography ( $n$-pentane- $\left.\mathrm{Et}_{2} \mathrm{O} 1: 2\right)$ gave 1d $(0.79 \mathrm{~g}$, $98 \%$ ) as a colorless solid; $\mathrm{mp} 91-93{ }^{\circ} \mathrm{C} ; R_{f}=0.4$ (n-pentane- $\mathrm{Et}_{2} \mathrm{O}$, $1: 2)$.

IR (ATR): 3278, 3144, 3092, 1655, 1593, 1493, 1447, 1280, 1207, $1124,874,836,745,690 \mathrm{~cm}^{-1}$.

${ }^{1} \mathrm{H}$ NMR $\left(600 \mathrm{MHz}, \mathrm{CDCl}_{3}\right): \delta=3.85\left(\mathrm{~s}, 3 \mathrm{H}, \mathrm{OCH}_{3}\right), 3.88(\mathrm{~s}, 3 \mathrm{H}$, $\left.\mathrm{OCH}_{3}\right), 6.49\left(\mathrm{~s}, 1 \mathrm{H}, \mathrm{CH}_{\mathrm{Ar}}\right), 6.57\left(\mathrm{~d}, J=9 \mathrm{~Hz}, 1 \mathrm{H}, \mathrm{CH}_{\mathrm{Ar}}\right), 7.17(\mathrm{t}$, $\left.J=7.2 \mathrm{~Hz}, 1 \mathrm{H}, \mathrm{CH}_{\mathrm{Ar}}\right), 7.38\left(\mathrm{t}, J=7.8 \mathrm{~Hz}, 2 \mathrm{H}, \mathrm{CH}_{\mathrm{Ar}}\right), 7.67(\mathrm{~d}$, $\left.J=7.8 \mathrm{~Hz}, 2 \mathrm{H}, \mathrm{CH}_{\mathrm{Ar}}\right), 7.92\left(\mathrm{dd}, J=1.8,8.4 \mathrm{~Hz}, 1 \mathrm{H}, \mathrm{CH}_{\mathrm{Ar}}\right), 8.53$ (br s, $1 \mathrm{H}, \mathrm{NH}$ ).

${ }^{13} \mathrm{C}$ NMR $\left(150 \mathrm{MHz}, \mathrm{CDCl}_{3}\right): \delta=55.7\left(\mathrm{OCH}_{3}\right), 56.1\left(\mathrm{OCH}_{3}\right), 98.9$ $\left(\mathrm{CH}_{\mathrm{Ar}}\right), 105.4\left(\mathrm{CH}_{\mathrm{Ar}}\right), 116.8(\mathrm{C}), 119.8\left(2 \mathrm{C}, \mathrm{CH}_{\mathrm{Ar}}\right), 124.9\left(\mathrm{CH}_{\mathrm{Ar}}\right)$, $129.1\left(2 \mathrm{C}, \mathrm{CH}_{\mathrm{Ar}}\right), 134.3\left(\mathrm{CH}_{\mathrm{Ar}}\right), 137.1(\mathrm{C}), 160.9(\mathrm{C}), 162.2(\mathrm{C})$, 165.7 (NCO), $188.5(\mathrm{CO})$.

MS (EI, $70 \mathrm{eV}): m / z(\%)=308.09\left([\mathrm{M}+\mathrm{Na}]^{+}\right), 286.11\left([\mathrm{M}+\mathrm{H}]^{+}\right)$. Anal. Calcd for $\mathrm{C}_{16} \mathrm{H}_{15} \mathrm{NO}_{2}$ : C, 67.36; H, 5.30; N, 4.91. Found: $\mathrm{C}$, $67.11 ; \mathrm{H}, 5.43 ; \mathrm{N}, 4.89$. 


\section{o-Methylmandelic Acid}

To a solution of 2-methylbenzaldehyde (12 g, $100 \mathrm{mmol}, 1$ equiv) and $\mathrm{BnEt}_{3} \mathrm{NCl}\left(1.23 \mathrm{~g}, 5 \mathrm{mmol}, 0.05\right.$ equiv) in $\mathrm{CHCl}_{3}(16 \mathrm{~mL})$ was added carefully aq $\mathrm{NaOH}(1 \mathrm{~g} / \mathrm{mL})(25 \mathrm{~mL})$. The resulting orange slurry was refluxed for $1 \mathrm{~h}$ and then cooled to r.t. $\mathrm{H}_{2} \mathrm{O}$ was added $(400 \mathrm{~mL})$ and the mixture was extracted with $\mathrm{Et}_{2} \mathrm{O}(2 \times 100 \mathrm{~mL})$. The remaining aqueous layer was acidified with concd $\mathrm{HCl}$ until $\mathrm{pH} 1$ and extracted with $\mathrm{Et}_{2} \mathrm{O}(6 \times 50 \mathrm{~mL})$. The combined organic layers were dried $\left(\mathrm{MgSO}_{4}\right)$, and the solvent removed in vacuo. $\mathrm{Re}$ crystallization (toluene) gave $o$-methylmandelic acid $(6.99 \mathrm{~g}, 42 \%)$ as a colorless solid; $R_{f}=0.27$ ( $n$-pentane- $\mathrm{Et}_{2} \mathrm{O}, 1: 1$ ).

${ }^{1} \mathrm{H}$ NMR (600 MHz, $\left.\mathrm{CDCl}_{3}\right): \delta=2.35\left(\mathrm{~s}, 3 \mathrm{H}, \mathrm{CH}_{3}\right), 5.19(\mathrm{~s}, 1 \mathrm{H}$, $\mathrm{C} H \mathrm{OH}), 7.15-7.27\left(\mathrm{~m}, 4 \mathrm{H}, \mathrm{CH}_{\mathrm{Ar}}\right)$.

${ }^{13} \mathrm{C} \mathrm{NMR}\left(150 \mathrm{MHz}, \mathrm{CDCl}_{3}\right): \delta=21.3\left(\mathrm{CH}_{3}\right), 73.0(\mathrm{CHOH}), 124.1$ $\left(\mathrm{CH}_{\mathrm{Ar}}\right), 127.5\left(\mathrm{CH}_{\mathrm{Ar}}\right), 129.0\left(\mathrm{CH}_{\mathrm{Ar}}\right), 130.0\left(\mathrm{CH}_{\mathrm{Ar}}\right), 137.6(\mathrm{C}), 138.9$ (C), $178.3(\mathrm{COOH})$.

\section{2-Methylphenylglyoxylic Acid}

$o$-Methylmandelic acid $(2.98 \mathrm{~g}, 0.025 \mathrm{~mol})$ was added to $1.0 \mathrm{M}$ $\mathrm{KOH}(75 \mathrm{~mL})$ and the mixture was stirred at $0{ }^{\circ} \mathrm{C}$ for $10 \mathrm{~min} . \mathrm{KM}$ $\mathrm{nO}_{4}(6.0 \mathrm{~g}, 37.8 \mathrm{mmol})$ was added portionwise and the resulting solution was stirred for $30 \mathrm{~min}$ at $0{ }^{\circ} \mathrm{C} . \mathrm{Na}_{2} \mathrm{SO}_{3}(21 \mathrm{~g}, 165 \mathrm{mmol})$ was added, and concd $\mathrm{HCl}$ was added carefully until the solution became colorless. The product was extracted with $\mathrm{CH}_{2} \mathrm{Cl}_{2}(4 \times 50$ $\mathrm{mL})$ and dried $\left(\mathrm{MgSO}_{4}\right)$. Evaporation of the solvent gave 2-methylphenylglyoxylic acid $(1.96 \mathrm{~g}, 48 \%)$ as a colorless solid; mp $73^{\circ} \mathrm{C}$; $R_{f}=0.5$ (n-pentane-acetone, $\left.1: 1\right)$.

IR (ATR): 2998 (s), 2661 (w), 2542 (w), 1679 (s), 1610 (m), 1417 (s), $1273(\mathrm{~s}), 1209(\mathrm{~m}), 1156(\mathrm{~m}), 1095(\mathrm{~s}), 1039(\mathrm{w}), 925(\mathrm{~m}), 841$ (m), $781(\mathrm{~m}), 724(\mathrm{~s}), 686 \mathrm{~cm}^{-1}(\mathrm{~m})$.

${ }^{1} \mathrm{H} \mathrm{NMR}\left(600 \mathrm{MHz}, \mathrm{CDCl}_{3}\right): \delta=2.44\left(\mathrm{~s}, 3 \mathrm{H}, \mathrm{CH}_{3}\right), 7.42(\mathrm{t}, J=7.2$ $\left.\mathrm{Hz}, 1 \mathrm{H}, \mathrm{CH}_{\mathrm{Ar}}\right), 7.51\left(\mathrm{~d}, 1 \mathrm{H}_{\mathrm{r}}, J=7.8 \mathrm{~Hz}, \mathrm{CH}_{\mathrm{A}}\right), 8.07\left(\mathrm{~m}, 2 \mathrm{H}, \mathrm{CH}_{\mathrm{Ar}}\right)$, 8.90 (br s, $1 \mathrm{H}, \mathrm{COOH})$.

${ }^{13} \mathrm{C}$ NMR $\left(150 \mathrm{MHz}, \mathrm{CDCl}_{3}\right): \delta=21.2\left(\mathrm{CH}_{3}\right), 128.4\left(\mathrm{CH}_{\mathrm{Ar}}\right), 128.9$ $\left(\mathrm{CH}_{\mathrm{Ar}}\right), 131.3\left(\mathrm{CH}_{\mathrm{Ar}}\right), 131.7(\mathrm{C}), 136.5\left(\mathrm{CH}_{\mathrm{Ar}}\right), 139.0(\mathrm{C}), 163.3$ $(\mathrm{COOH}), 184.9(\mathrm{COCOOH})$.

MS (EI, $70 \mathrm{eV}): m / z(\%)=164(5), 120$ (7), 119 (100), 91 (45), 65 (10).

Anal. Calcd for $\mathrm{C}_{9} \mathrm{H}_{8} \mathrm{O}_{3}$ : C, 65.85; H, 4.91. Found: C, 65.51; H, 4.97.

\section{2-Oxo- $N$-phenyl-2-(2-tolyl)acetamide (1e)}

Following the general procedure using aniline $(1.56 \mathrm{~g}, 16.8 \mathrm{mmol}$, 1.4 equiv) and 2-methylphenylglyoxylic acid (1.96 g, $12 \mathrm{mmol}, 1$ equiv), with purification of the crude product by flash column chromatography ( $n$-pentane- $\left.\mathrm{Et}_{2} \mathrm{O}, 6: 1\right)$ gave $1 \mathrm{e}(1.64 \mathrm{~g}, 57 \%)$ as a yellow solid; $\mathrm{mp} 82{ }^{\circ} \mathrm{C} ; R_{f}=0.39$ (n-pentane- $\mathrm{Et}_{2} \mathrm{O}, 6: 1$ ).

IR (ATR): 3360, 3054, 2913, 1686, 1656, 1595, 1535, 1494, 1439, $1375,1281,1150,1105,1037,983,894,825,744,680 \mathrm{~cm}^{-1}$.

${ }^{1} \mathrm{H}$ NMR $\left(600 \mathrm{MHz}, \mathrm{CDCl}_{3}\right): \delta=2.43\left(\mathrm{~s}, 3 \mathrm{H}, \mathrm{CH}_{3}\right), 7.19(\mathrm{t}, J=7.2$ $\left.\mathrm{Hz}, 1 \mathrm{H}, \mathrm{CH}_{\mathrm{Ar}}\right), 7.39\left(\mathrm{~m}, 3 \mathrm{H}, \mathrm{CH}_{\mathrm{Ar}}\right), 7.47\left(\mathrm{~d}, J=7.2 \mathrm{~Hz}, 1 \mathrm{H}, \mathrm{CH}_{\mathrm{Ar}}\right)$, $7.70\left(\mathrm{~d}, J=7.8 \mathrm{~Hz}, 2 \mathrm{H}, \mathrm{CH}_{\mathrm{Ar}}\right), 8.21\left(\mathrm{~m}, 2 \mathrm{H}, \mathrm{CH}_{\mathrm{Ar}}\right), 8.94(\mathrm{br} \mathrm{s}, 1 \mathrm{H}$, $\mathrm{NH})$.

${ }^{13} \mathrm{C} \mathrm{NMR}\left(150 \mathrm{MHz}, \mathrm{CDCl}_{3}\right): \delta=21.3\left(\mathrm{CH}_{3}\right), 119.9\left(\mathrm{CH}_{\mathrm{Ar}}\right), 125.3$ $\left(\mathrm{CH}_{\mathrm{Ar}}\right), 128.5\left(\mathrm{CH}_{\mathrm{Ar}}\right), 128.7\left(\mathrm{CH}_{\mathrm{Ar}}\right), 129.2\left(\mathrm{CH}_{\mathrm{Ar}}\right), 131.8\left(\mathrm{CH}_{\mathrm{Ar}}\right)$ 133.0 (C), $135.5\left(\mathrm{CH}_{\mathrm{Ar}}\right), 136.7$ (C), 138.4 (C), 159.0 (NCO), 187.6 (CO).

MS (EI, $70 \mathrm{eV}): m / z(\%)=239$ (31), 120 (12), 119 (100), 91 (31), 65 (10).

Anal. Calcd for $\mathrm{C}_{15} \mathrm{H}_{13} \mathrm{NO}_{2}$ : C, 75.30; H, 5.48; N, 5.85. Found: C, 75.33; H, 5.48; N, 6.02 .

\section{2-Oxo-2-phenyl- $\mathrm{N}$-tosylacetamide (1f)}

$\mathrm{SOCl}_{2}(0.6 \mathrm{~mL}, 8 \mathrm{mmol}, 1.2$ equiv) was added dropwise to a solution of phenylglyoxylic acid ( $1 \mathrm{~g}, 6.6 \mathrm{mmol}, 1$ equiv) in $N, N$-di- methylacetamide $(16 \mathrm{~mL})$ cooled to $0{ }^{\circ} \mathrm{C}$. After stirring at this temperature for $10 \mathrm{~min}, \mathrm{TsNH}_{2}(1.61 \mathrm{~g}, 9.4 \mathrm{mmol}, 1.4$ equiv) was added and the mixture was stirred at $60{ }^{\circ} \mathrm{C}$ overnight, then poured into a mixture of ice and $\mathrm{H}_{2} \mathrm{O}$ and stirred at r.t. for $4.5 \mathrm{~h}$. The product was extracted with $\mathrm{Et}_{2} \mathrm{O}(3 \times 15 \mathrm{~mL})$, the combined organic layers were washed with $\mathrm{H}_{2} \mathrm{O}$ and brine, dried $\left(\mathrm{Na}_{2} \mathrm{SO}_{4}\right)$, and concentrated in vacuo. The crude product was purified by flash column chromatography ( $n$-pentane- $\left.\mathrm{Et}_{2} \mathrm{O}, 1: 1\right)$ to afford $\mathbf{1 f}(0.82 \mathrm{~g}, 41 \%)$ as a colorless solid; $\mathrm{mp} 52-55{ }^{\circ} \mathrm{C} ; R_{f}=0.43$ (EtOAc).

IR (ATR): 3357, 3253, 1717, 1680, 1594, 1527, 1491, 1410, 1346, $1298,1269,1188,1167,1132,1087,977,874,813,775,739,662$ $\mathrm{cm}^{-1}$.

${ }^{1} \mathrm{H}$ NMR (600 MHz, $\left.\mathrm{CD}_{3} \mathrm{OD}\right): \delta=2.44\left(\mathrm{~s}, 3 \mathrm{H}, \mathrm{CH}_{3}\right), 7.34(\mathrm{~d}$, $\left.J=7.8 \mathrm{~Hz}, 2 \mathrm{H}, \mathrm{CH}_{\mathrm{Ar}}\right), 7.45\left(\mathrm{t}, J=7.8 \mathrm{~Hz}, 2 \mathrm{H}, \mathrm{CH}_{\mathrm{Ar}}\right), 7.61(\mathrm{t}$, $\left.J=7.2 \mathrm{~Hz}, 1 \mathrm{H}, \mathrm{CH}_{\mathrm{Ar}}\right), 7.87\left(\mathrm{~d}, J=7.2 \mathrm{~Hz}, 2 \mathrm{H}, \mathrm{CH}_{\mathrm{Ar}}\right), 7.91(\mathrm{~d}$, $\left.J=7.8 \mathrm{~Hz}, 2 \mathrm{H}, \mathrm{CH}_{\mathrm{Ar}}\right)$.

${ }^{13} \mathrm{C} \mathrm{NMR}\left(150 \mathrm{MHz}, \mathrm{CD}_{3} \mathrm{OD}\right): \delta=20.0\left(\mathrm{CH}_{3}\right), 126.8\left(2 \mathrm{C}, \mathrm{CH}_{\mathrm{Ar}}\right)$, $128.2\left(2 \mathrm{C}, \mathrm{CH}_{\mathrm{Ar}}\right), 128.5\left(2 \mathrm{C}, \mathrm{CH}_{\mathrm{Ar}}\right), 129.5\left(2 \mathrm{C}, \mathrm{CH}_{\mathrm{Ar}}\right), 133.4(\mathrm{C})$, $133.6\left(\mathrm{CH}_{\mathrm{Ar}}\right), 140.4(\mathrm{C}), 142.2(\mathrm{C}), 172.3(\mathrm{NCO}), 192.9(\mathrm{CO})$.

MS (ESI): $m / z(\%)=326\left(100,[\mathrm{M}+\mathrm{Na}]^{+}\right)$.

HRMS: $m / z[\mathrm{M}+\mathrm{Na}]^{+}$calcd for $\mathrm{C}_{15} \mathrm{H}_{13} \mathrm{NO}_{4} \mathrm{NaS}$ : 326.0457; found: 326.0457 .

\section{Ethyl (E)-3-[(2S,3R,4R)-4-Hydroxy-5-oxo-1,2,4-triphenylpyr-} rolidin-3-yl]acrylate (6a)

Following the general procedure with purification by flash chromatography (n-pentane-EtOAc, 3:1) gave 6a as a colorless solid; yield: $91 \mathrm{mg}(65 \%) ; \mathrm{mp} 165-170{ }^{\circ} \mathrm{C} ; 87 \%$ ee [chiral stationary phase HPLC (Daicel AS)]; $R_{f}=0.46(n$-pentane-EtOAc, 3:1); $[\alpha]_{\mathrm{D}}^{22}+109.0\left(\mathrm{c} 0.51, \mathrm{CHCl}_{3}\right)$.

IR (ATR): 3336, 3054, 2984, 1696, 1601, 1538, 1495, 1449, 1374, $1276,1174,1120,1033,979,871,749,693 \mathrm{~cm}^{-1}$.

${ }^{1} \mathrm{H}$ NMR $\left(600 \mathrm{MHz}, \mathrm{CDCl}_{3}\right): \delta=1.24\left(\mathrm{t}, J=7.2 \mathrm{~Hz}, 3 \mathrm{H}, \mathrm{CH}_{3}\right)$, $2.95(\mathrm{t}, J=8.4 \mathrm{~Hz}, 1 \mathrm{H}, \mathrm{NCHCH}), 3.46(\mathrm{br} \mathrm{s}, 1 \mathrm{H}, \mathrm{OH}), 4.13(\mathrm{q}$, $\left.J=7.2 \mathrm{~Hz}, 2 \mathrm{H}, \mathrm{CH}_{2}\right), 5.24(\mathrm{~d}, J=8.4 \mathrm{~Hz}, 1 \mathrm{H}, \mathrm{NCHPh}), 5.36$ (d, $J=15 \mathrm{~Hz}, 1 \mathrm{H}, \mathrm{CHCOOEt}), 7.08-7.47\left(\mathrm{~m}, 16 \mathrm{H}, \mathrm{CH}_{\mathrm{Ar}}, \mathrm{CH}=\mathrm{CH}-\right.$ COOEt).

${ }^{13} \mathrm{C} \mathrm{NMR}\left(150 \mathrm{MHz}, \mathrm{CDCl}_{3}\right): \delta=14.1\left(\mathrm{CH}_{3}\right), 59.7(\mathrm{NCHCH}), 60.5$ $\left(\mathrm{CH}_{2}\right), 65.1(\mathrm{NCHPh}), 80.8(\mathrm{COH}), 123.8\left(2 \mathrm{C}, \mathrm{CH}_{\mathrm{Ar}}\right), 125.8(2 \mathrm{C}$, $\left.\mathrm{CH}_{\mathrm{Ar}}\right), 125.9\left(\mathrm{CH}_{\mathrm{Ar}}\right), 126.4(\mathrm{CHCOOEt}), 127.0\left(2 \mathrm{C}, \mathrm{CH}_{\mathrm{Ar}}\right), 128.1$ $\left(\mathrm{CH}_{\mathrm{Ar}}\right), 128.2\left(\mathrm{CH}_{\mathrm{Ar}}\right), 128.4\left(2 \mathrm{C}, \mathrm{CH}_{\mathrm{Ar}}\right), 128.6\left(2 \mathrm{C}, \mathrm{CH}_{\mathrm{Ar}}\right), 128.8$ $\left(2 \mathrm{C}, \mathrm{CH}_{\mathrm{Ar}}\right), 137.0(\mathrm{C}), 137.2(\mathrm{C}), 139.7(\mathrm{CH}=\mathrm{CHCOOEt}), 140.5$ (C), 165.5 (COOEt), 173.8 (NCO).

MS (EI, $70 \mathrm{eV}): m / z(\%)=428$ (19), 427 (60), 410 (13), 409 (43), 335 (11), 203 (21), 202 (34), 182 (42), 181 (17), 180 (21), 157 (36), 144 (11), 129 (39), 128 (22), 115 (12), 105 (100), 104 (10), 91 (13), 77 (53).

Anal. Calcd for $\mathrm{C}_{27} \mathrm{H}_{25} \mathrm{NO}_{4}$ : C, 75.86; H, 5.89; N, 3.28. Found: C, $75.61 ; \mathrm{H}, 5.87 ; \mathrm{N}, 3.14$.

\section{Ethyl $(E)-3-[(2 S, 3 R, 4 R)-4-H y d r o x y-2-(4-m e t h o x y p h e n y l)-5-$ oxo-1,4-diphenylpyrrolidin-3-yl] acrylate (6b)}

Following the general procedure with purification by flash chromatography (n-pentane-EtOAc, 3:1) gave $\mathbf{6 b}$ as a colorless solid; yield: $81 \mathrm{mg}(54 \%) ; \mathrm{mp} 85-88^{\circ} \mathrm{C} ; 95 \%$ ee [chiral stationary phase HPLC (Daicel AS)]; $R_{f}=0.29\left(n\right.$-pentane-EtOAc, 3:1); $[\alpha]_{\mathrm{D}}^{22}$ $+118.6\left(\right.$ c $\left.0.5, \mathrm{CHCl}_{3}\right)$.

IR (ATR): 3357, 2976, 1699, 1603, 1506, 1373, 1247, 1175, 1115, $1031,979,831,751,692 \mathrm{~cm}^{-1}$.

${ }^{1} \mathrm{H}$ NMR $\left(600 \mathrm{MHz}, \mathrm{CDCl}_{3}\right): \delta=1.24\left(\mathrm{t}, J=7.2 \mathrm{~Hz}, 3 \mathrm{H}, \mathrm{CH}_{3}\right)$, 2.94 (t, $J=9 \mathrm{~Hz}, 1 \mathrm{H}, \mathrm{NCHCH}), 3.24$ (br s, $1 \mathrm{H}, \mathrm{OH}), 2.72$ (s, $3 \mathrm{H}$, $\left.\mathrm{OCH}_{3}\right), 4.14\left(\mathrm{q}, J=7.2 \mathrm{~Hz}, 2 \mathrm{H}, \mathrm{CH}_{2}\right), 5.20(\mathrm{~d}, J=9 \mathrm{~Hz}, 1 \mathrm{H}$, $\mathrm{NCHPh}), 5.39$ (d, $J=15.6 \mathrm{~Hz}, 1 \mathrm{H}, \mathrm{CHCOOEt}), 6.75(\mathrm{~d}, J=9 \mathrm{~Hz}$, $\left.2 \mathrm{H}, \mathrm{CH}_{\mathrm{Ar}}\right), 7.08-7.12\left(\mathrm{~m}, 3 \mathrm{H}, \mathrm{CH}_{\mathrm{Ar}}\right), 7.20(\mathrm{dd}, J=9,15.6 \mathrm{~Hz}, 1 \mathrm{H}$, $\mathrm{CH}=\mathrm{CHCOOEt}), 7.24-7.49\left(\mathrm{~m}, 9 \mathrm{H}, \mathrm{CH}_{\mathrm{Ar}}\right)$. 
${ }^{13} \mathrm{C}$ NMR $\left(150 \mathrm{MHz}, \mathrm{CDCl}_{3}\right): \delta=14.2\left(\mathrm{CH}_{3}\right), 55.1\left(\mathrm{OCH}_{3}\right), 59.7$ $(\mathrm{NCHCH}), 60.5\left(\mathrm{CH}_{2}\right), 64.7(\mathrm{NCHPh}), 80.8(\mathrm{COH}), 124.0(2 \mathrm{C}$ $\left.\mathrm{CH}_{\mathrm{Ar}}\right), 125.8\left(2 \mathrm{C}, \mathrm{CH}_{\mathrm{Ar}}\right), 125.9(\mathrm{CHCOOEt}), 126.4\left(\mathrm{CH}_{\mathrm{Ar}}\right), 128.2$ $\left(\mathrm{CH}_{\mathrm{Ar}}\right), 128.3\left(2 \mathrm{C}, \mathrm{CH}_{\mathrm{Ar}}\right), 128.5\left(2 \mathrm{C}, \mathrm{CH}_{\mathrm{Ar}}\right), 128.6\left(2 \mathrm{C}, \mathrm{CH}_{\mathrm{Ar}}\right)$ $128.9(\mathrm{C}), 137.0(\mathrm{C}), 139.8$ (CH=CHCOOEt), $140.6(\mathrm{C}), 159.3(\mathrm{C})$, 165.5 (COOEt), 173.8 (NCO).

MS (EI, $70 \mathrm{eV}): m / z(\%)=457(14), 440(32), 439$ (100), $365(15)$ 232 (20), 212 (45), 211 (44), 210 (23), 187 (17), 159 (14), 121 (41), 105 (41), 77 (25).

HRMS: $m / z[\mathrm{M}+\mathrm{H}]^{+}$calcd for $\mathrm{C}_{28} \mathrm{H}_{28} \mathrm{NO}_{5}$ : 458.1962; found: 458.1962 .

\section{Ethyl $(E)-3-[(2 S, 3 R, 4 R)-2-(4-C h l o r o p h e n y l)-4-h y d r o x y-5-0 x o-$} 1,4-diphenylpyrrolidin-3-yl]acrylate (6c)

Following the general procedure with purification by flash chromatography (n-pentane-EtOAc, 3:1) gave $\mathbf{6 c}$ as a colorless solid; yield: $88 \mathrm{mg}(58 \%) ; \mathrm{mp} 90-92{ }^{\circ} \mathrm{C} ; 90 \%$ ee [chiral stationary phase HPLC (Daicel AS)]; $R_{f}=0.4$ (n-pentane-EtOAc, 3:1); $[\alpha]_{\mathrm{D}}^{22}$ $+127.1\left(c 0.5, \mathrm{CHCl}_{3}\right)$.

IR (ATR): 3362, 1699, 1596, 1494, 1452, 1373, 1253, 1174, 1090, $1027,981,832,797,749,692 \mathrm{~cm}^{-1}$.

${ }^{1} \mathrm{H}$ NMR $\left(600 \mathrm{MHz}, \mathrm{CDCl}_{3}\right): \delta=1.24\left(\mathrm{t}, J=7.2 \mathrm{~Hz}, 3 \mathrm{H}, \mathrm{CH}_{3}\right)$, $2.86(\mathrm{t}, J=8.8 \mathrm{~Hz}, 1 \mathrm{H}, \mathrm{NCHCH}), 3.59$ (br s, $1 \mathrm{H}, \mathrm{OH}), 4.14(\mathrm{q}$ $\left.J=7.2 \mathrm{~Hz}, 2 \mathrm{H}, \mathrm{CH}_{2}\right), 5.22(\mathrm{~d}, J=8.4 \mathrm{~Hz}, 1 \mathrm{H}, \mathrm{NC} H \mathrm{Ph}), 5.35(\mathrm{~d}$, $J=16 \mathrm{~Hz}, 1 \mathrm{H}, \mathrm{CHCOOEt}), 7.08-7.43(\mathrm{~m}, 15 \mathrm{H}, \mathrm{CH}=\mathrm{CHCOOEt}$, $\mathrm{CH}_{\mathrm{Ar}}$ ).

${ }^{13} \mathrm{C}$ NMR $\left(150 \mathrm{MHz}, \mathrm{CDCl}_{3}\right): \delta=14.1\left(\mathrm{CH}_{3}\right), 59.8(\mathrm{NCHCH}), 60.6$ $\left(\mathrm{CH}_{2}\right), 64.5(\mathrm{NCHPh}), 80.7(\mathrm{COH}), 123.7\left(2 \mathrm{C}, \mathrm{CH}_{\mathrm{Ar}}\right), 125.8(2 \mathrm{C}$ $\left.\mathrm{CH}_{\mathrm{Ar}}\right), 126.1\left(\mathrm{CH}_{\mathrm{Ar}}\right), 126.7(\mathrm{CHCOOEt}), 128.2\left(\mathrm{CH}_{\mathrm{Ar}}\right), 128.4(2 \mathrm{C}$ $\left.\mathrm{CH}_{\mathrm{Ar}}\right), 128.5\left(2 \mathrm{C}, \mathrm{CH}_{\mathrm{Ar}}\right), 128.8\left(2 \mathrm{C}, \mathrm{CH}_{\mathrm{Ar}}\right), 129.1\left(2 \mathrm{C}, \mathrm{CH}_{\mathrm{Ar}}\right)$, $134.0(\mathrm{C}), 135.8(\mathrm{C}), 136.8(\mathrm{C}), 139.3(\mathrm{CH}=\mathrm{CHCOOEt}), 140.2(\mathrm{C})$, 165.4 (COOEt), 173.8 (NCO).

MS (EI, $70 \mathrm{eV}): m / z(\%)=461$ (23), 443 (34), 346 (23), 345 (18), 344 (48), 258 (18), 243 (25), 236 (21), 216 (29), 191 (17), 105 (100), 77 (60).

HRMS: $m / z[\mathrm{M}+\mathrm{H}]^{+}$calcd for $\mathrm{C}_{27} \mathrm{H}_{25} \mathrm{NO}_{4} \mathrm{Cl}$ : 462.1467; found: 462.1467 .

\section{Ethyl (E)-3-\{(2S,3R,4R)-2-[4-(Dimethylamino)phenyl]-4-hy-} droxy-5-oxo-1,4-diphenylpyrrolidin-3-yl\}acrylate (6d) Following the general procedure with purification by flash chromatography (n-pentane-EtOAc, 3:1) gave $\mathbf{6 d}$ as a colorless solid; yield: $31 \mathrm{mg}(20 \%) ; \mathrm{mp} 89-91{ }^{\circ} \mathrm{C} ; 89 \%$ ee [chiral stationary phase HPLC (Daicel AS)]; $R_{f}=0.26(n$-pentane-EtOAc, $3: 1) ;[\alpha]_{\mathrm{D}}{ }^{22}$ $+79.5\left(\right.$ c $\left.1.0, \mathrm{CHCl}_{3}\right)$.

IR (ATR): 3356, 2981, 2919, 2805, 1699, 1611, 1522, 1451, 1364, $1172,1124,1038,980,868,815,749,692 \mathrm{~cm}^{-1}$.

${ }^{1} \mathrm{H}$ NMR $\left(600 \mathrm{MHz}, \mathrm{CDCl}_{3}\right): \delta=1.24\left(\mathrm{t}, J=7.2 \mathrm{~Hz}, 3 \mathrm{H}, \mathrm{CH}_{3}\right)$, $2.87\left[\mathrm{~s}, 6 \mathrm{H}, \mathrm{N}\left(\mathrm{CH}_{3}\right)_{2}\right], 2.97$ (t, $\left.J=9.0 \mathrm{~Hz}, 1 \mathrm{H}, \mathrm{NCHCH}\right), 3.40(\mathrm{br}$ $\mathrm{s}, 1 \mathrm{H}, \mathrm{OH}), 4.13\left(\mathrm{q}, J=7.2 \mathrm{~Hz}, 2 \mathrm{H}, \mathrm{CH}_{2}\right), 5.15(\mathrm{~d}, J=8.4 \mathrm{~Hz}, 1 \mathrm{H}$ $\mathrm{NCHPh}), 5.41(\mathrm{~d}, J=16.2 \mathrm{~Hz}, 1 \mathrm{H}, \mathrm{CHCOOEt}), 6.55(\mathrm{~m}, 2 \mathrm{H}$, $\left.\mathrm{CH}_{\mathrm{Ar}}\right), 7.01\left(\mathrm{~d}, J=8.4 \mathrm{~Hz}, 2 \mathrm{H}, \mathrm{CH}_{\mathrm{Ar}}\right), 7.09(\mathrm{t}, J=7.2 \mathrm{~Hz}, 1 \mathrm{H}$ $\mathrm{CH}_{\mathrm{Ar}}$ ), 7.18 (dd, $J=9,16.2 \mathrm{~Hz}, 1 \mathrm{H}, \mathrm{C} H=\mathrm{CHCOOEt}$ ), 7.22-7.33 $\left(\mathrm{m}, 5 \mathrm{H}, \mathrm{CH}_{\mathrm{Ar}}\right), 7.39\left(\mathrm{t}, J=7.8 \mathrm{~Hz}, 2 \mathrm{H}, \mathrm{CH}_{\mathrm{Ar}}\right), 7.48(\mathrm{~d}, J=8.4 \mathrm{~Hz}$, $\left.2 \mathrm{H}, \mathrm{CH}_{\mathrm{Ar}}\right)$.

${ }^{13} \mathrm{C} \mathrm{NMR}\left(150 \mathrm{MHz}, \mathrm{CDCl}_{3}\right): \delta=14.2\left(\mathrm{CH}_{3}\right), 40.3\left[\mathrm{~N}\left(\mathrm{CH}_{3}\right)_{2}\right], 59.6$ $(\mathrm{NCHCH}), 60.4\left(\mathrm{CH}_{2}\right), 64.9(\mathrm{NCHPh}), 80.9(\mathrm{COH}), 112.3(2 \mathrm{C}$, $\left.\mathrm{CH}_{\mathrm{Ar}}\right), 124.1\left(2 \mathrm{C}, \mathrm{CH}_{\mathrm{Ar}}\right), 125.8\left(\mathrm{CH}_{\mathrm{Ar}}\right), 125.9\left(2 \mathrm{C}, \mathrm{CH}_{\mathrm{Ar}}\right), 126.1$ (CHCOOEt), $128.0\left(2 \mathrm{C}, \mathrm{CH}_{\mathrm{Ar}}\right), 128.1\left(2 \mathrm{C}, \mathrm{CH}_{\mathrm{Ar}}\right), 128.4(2 \mathrm{C}$, $\left.\mathrm{CH}_{\mathrm{Ar}}\right), 128.5\left(\mathrm{CH}_{\mathrm{Ar}}\right), 137.2(2 \mathrm{C}, \mathrm{C}), 140.2(\mathrm{CH}=\mathrm{CHCOOEt}), 140.9$ (2 C, C), 165.6 (COOEt), 173.8 (NCO).

MS (EI, $70 \mathrm{eV}): m / z(\%)=471(20), 470(61), 401(14), 400(53)$, 267 (17), 252 (23), 245 (19), 225 (31), 224 (100), 222 (47), 176 (26), 175 (22), 174 (10), 172 (17), 134 (53), 121 (11), 105 (44), 77 (24).
HRMS: $m / z[\mathrm{M}+\mathrm{H}]^{+}$calcd for $\mathrm{C}_{29} \mathrm{H}_{31} \mathrm{~N}_{2} \mathrm{O}_{4}$ : 471.2278; found: 471.2278 .

\section{Ethyl (E)-3-[(2S,3R,4R)-4-Hydroxy-2-(4-nitrophenyl)-5-0x0-} 1,4-diphenylpyrrolidin-3-yl]acrylate (6e)

Following the general procedure with purification by flash chromatography (n-pentane-EtOAc, 3:1) gave $\mathbf{6 e}$ as a colorless solid; yield: $33 \mathrm{mg}(21 \%) ; \mathrm{mp} 98-100{ }^{\circ} \mathrm{C}$; $86 \%$ ee [chiral stationary phase HPLC (Daicel AS)]; $R_{f}=0.23$ (n-pentane-EtOAc, 3:1); $[\alpha]_{\mathrm{D}}{ }^{22}$ $+114.5\left(c 0.25, \mathrm{CHCl}_{3}\right)$

IR (ATR): 3365, 3071, 2929, 1701, 1599, 1520, 1449, 1346, 1248, $1173,1109,1130,979,855,796,748,691 \mathrm{~cm}^{-1}$.

${ }^{1} \mathrm{H}$ NMR $\left(600 \mathrm{MHz}, \mathrm{CDCl}_{3}\right): \delta=1.25\left(\mathrm{t}, J=7.2 \mathrm{~Hz}, 3 \mathrm{H}, \mathrm{CH}_{3}\right)$, $2.85(\mathrm{t}, J=9.0 \mathrm{~Hz}, 1 \mathrm{H}, \mathrm{NCHCH}), 3.56($ br s, $1 \mathrm{H}, \mathrm{OH}), 4.15(\mathrm{q}$, $\left.J=7.2 \mathrm{~Hz}, 2 \mathrm{H}, \mathrm{CH}_{2}\right), 5.34$ (d, $\left.J=15.6 \mathrm{~Hz}, 1 \mathrm{H}, \mathrm{CHCOOEt}\right), 5.39$ $(\mathrm{d}, J=8.4 \mathrm{~Hz}, 1 \mathrm{H}, \mathrm{NCHPh}), 7.14\left(\mathrm{t}, J=7.8 \mathrm{~Hz}, 1 \mathrm{H}, \mathrm{CH}_{\mathrm{Ar}}\right), 7.18$ (dd, $J=9.6,15.6 \mathrm{~Hz}, 1 \mathrm{H}, \mathrm{CH}=\mathrm{CHCOOEt}), 7.26-7.46(\mathrm{~m}, 11 \mathrm{H}$, $\left.\mathrm{CH}_{\mathrm{Ar}}\right), 8.11\left(\mathrm{~d}, J=9.0 \mathrm{~Hz}, 2 \mathrm{H}, \mathrm{CH}_{\mathrm{Ar}}\right)$.

${ }^{13} \mathrm{C} \mathrm{NMR}\left(150 \mathrm{MHz}, \mathrm{CDCl}_{3}\right): \delta=14.1\left(\mathrm{CH}_{3}\right), 59.8(\mathrm{NCHCH}), 60.7$ $\left(\mathrm{CH}_{2}\right), 64.4(\mathrm{NCHPh}), 80.7(\mathrm{COH}), 123.4\left(2 \mathrm{C}, \mathrm{CH}_{\mathrm{Ar}}\right), 124.2(2 \mathrm{C}$, $\left.\mathrm{CH}_{\mathrm{Ar}}\right), 125.8\left(2 \mathrm{C}, \mathrm{CH}_{\mathrm{Ar}}\right), 126.4\left(\mathrm{CH}_{\mathrm{Ar}}\right), 127.2(\mathrm{CHCOOEt}), 127.9$ $\left(2 \mathrm{C}, \mathrm{CH}_{\mathrm{Ar}}\right), 128.5\left(\mathrm{CH}_{\mathrm{Ar}}\right), 128.6\left(2 \mathrm{C}, \mathrm{CH}_{\mathrm{Ar}}\right), 129.0\left(2 \mathrm{C}, \mathrm{CH}_{\mathrm{Ar}}\right)$, $136.5(\mathrm{C}), 138.6(\mathrm{CH}=\mathrm{CHCOOEt}), 139.8(\mathrm{C}), 144.7$ (C), 147.7 (C), 165.2 (COOEt), 173.7 (NCO).

MS (EI, $70 \mathrm{eV}): m / z(\%)=473(14), 472$ (51), 455 (28), 454 (89), 437 (24), 357 (15), 355 (16), 339 (13), 248 (25), 227 (62), 226 (30), 225 (100), 220 (13), 202 (42), 105 (79).

HRMS: $m / z[\mathrm{M}+\mathrm{H}]^{+}$calcd for $\mathrm{C}_{27} \mathrm{H}_{25} \mathrm{~N}_{2} \mathrm{O}_{6}: 473.1707$; found: 473.1708.

\section{Ethyl (E)-3-\{(2S,3R,4R)-4-Hydroxy-5-oxo-1,4-diphenyl-2- [3,4,5-tris(benzyloxy)phenyl]pyrrolidin-3-yl\}acrylate (6f)} Following the general procedure with purification by flash chromatography ( $n$-pentane-EtOAc, 3:1) gave $6 \mathbf{f}$ as a colorless solid; yield: $144 \mathrm{mg}(52 \%)$; mp $55-57^{\circ} \mathrm{C} ; 82 \%$ ee [chiral stationary phase HPLC (Daicel AS)]; $R_{f}=0.33$ (n-pentane-EtOAc, 3:1); $[\alpha]_{\mathrm{D}}^{22}$ $+94.5\left(c 1.0, \mathrm{CHCl}_{3}\right)$.

IR (ATR): 3362, 3063, 3032, 1702, 1592, 1497, 1441, 1372, 1309, $1242,1216,1166,1103,1029,978,908,836,792,739,693 \mathrm{~cm}^{-1}$.

${ }^{1} \mathrm{H}$ NMR (600 MHz, DMSO- $\left.d_{6}\right): \delta=1.26$ (t, $\left.J=7.2 \mathrm{~Hz}, 3 \mathrm{H}, \mathrm{CH}_{3}\right)$, $2.90(\mathrm{t}, J=8.4 \mathrm{~Hz}, 1 \mathrm{H}, \mathrm{NCHCH}), 4.04(\mathrm{q}, J=7.2 \mathrm{~Hz}, 2 \mathrm{H}$, $\left.\mathrm{CH}_{2} \mathrm{CH}_{3}\right), 4.77\left(\mathrm{~m}, 2 \mathrm{H}, \mathrm{CH}_{2} \mathrm{Ph}\right), 4.95\left(\mathrm{~d}, J=12 \mathrm{~Hz}, 2 \mathrm{H}, \mathrm{CH}_{2} \mathrm{Ph}\right)$, $5.05\left(\mathrm{~d}, J=12.6 \mathrm{~Hz}, 2 \mathrm{H}, \mathrm{CH}_{2} \mathrm{Ph}\right), 5.26(\mathrm{~d}, J=15.6 \mathrm{~Hz}, 1 \mathrm{H}, \mathrm{CH}-$ COOEt), $5.36(\mathrm{~d}, J=16.2 \mathrm{~Hz}, 1 \mathrm{H}, \mathrm{NCHPh}), 6.75\left(\mathrm{~s}, 2 \mathrm{H}, \mathrm{CH}_{\mathrm{Ar}}\right.$ ), $6.81\left(\mathrm{~s}, 1 \mathrm{H}, \mathrm{CH}_{\mathrm{Ar}}\right), 7.00(\mathrm{dd}, J=8.4,15.6 \mathrm{~Hz}, 1 \mathrm{H}, \mathrm{CH}=\mathrm{CH}-$ COOEt), $7.10\left(\mathrm{~m}, 1 \mathrm{H}, \mathrm{CH}_{\mathrm{Ar}}\right), 7.20-7.49\left(\mathrm{~m}, 23 \mathrm{H}, \mathrm{CH}_{\mathrm{Ar}}\right)$.

${ }^{13} \mathrm{C}$ NMR $\left(150 \mathrm{MHz}, \mathrm{DMSO}-d_{6}\right): \delta=14.4\left(\mathrm{CH}_{3}\right), 59.3(\mathrm{NCHCH})$, $60.4\left(\mathrm{CH}_{2} \mathrm{CH}_{3}\right), 64.0(\mathrm{NCHPh}), 70.6\left(2 \mathrm{C}, \mathrm{CH}_{2} \mathrm{Ph}\right), 74.3\left(\mathrm{CH}_{2} \mathrm{Ph}\right)$, $80.4(\mathrm{COH}), 107.7\left(\mathrm{CH}_{\mathrm{Ar}}\right), 124.7\left(2 \mathrm{C}, \mathrm{CH}_{\mathrm{Ar}}\right), 125.4(\mathrm{CHCOOEt})$, $125.9\left(\mathrm{CH}_{\mathrm{Ar}}\right), 126.9\left(2 \mathrm{C}, \mathrm{CH}_{\mathrm{Ar}}\right), 127.9\left(\mathrm{CH}_{\mathrm{Ar}}\right), 128.0\left(4 \mathrm{C}, \mathrm{CH}_{\mathrm{Ar}}\right)$, $128.1\left(2 \mathrm{C}, \mathrm{CH}_{\mathrm{Ar}}\right), 128.2\left(2 \mathrm{C}, \mathrm{CH}_{\mathrm{Ar}}\right), 128.4\left(2 \mathrm{C}, \mathrm{CH}_{\mathrm{Ar}}\right), 128.5(2$ $\left.\mathrm{C}, \mathrm{CH}_{\mathrm{Ar}}\right), 128.7\left(2 \mathrm{C}, \mathrm{CH}_{\mathrm{Ar}}\right), 128.8\left(4 \mathrm{C}, \mathrm{CH}_{\mathrm{Ar}}\right), 133.7(\mathrm{C}), 137.1$ (C), 137.4 (2 C, C), 137.9 (C), 138.0 (C), 141.6 (C), 141.9 (CH=CHCOOEt), 152.3 (2 C, C), 165.2 (COOEt), 173.2 (NCO).

MS (EI, $70 \mathrm{eV}): m / z(\%)=746(18), 546$ (10), 391 (18), 390 (100), 363 (68), 282 (15), 241 (11), 227 (55), 181 (34).

HRMS: $m / z[\mathrm{M}+\mathrm{H}]^{+}$calcd for $\mathrm{C}_{48} \mathrm{H}_{44} \mathrm{NO}_{7}$ : 746.3112; found: 746.3130 .

Ethyl (E)-3-[(2S,3R,4R)-2-(1,3-Benzodioxol-5-yl)-4-hydroxy-5oxo-1,4-diphenylpyrrolidin-3-yl]acrylate (6g)

Following the general procedure with purification by flash chromatography (n-pentane-EtOAc, $3: 1)$ gave $\mathbf{6 g}$ as a colorless solid; yield: $87 \mathrm{mg}(56 \%) ; \mathrm{mp} 98-100{ }^{\circ} \mathrm{C} ; 89 \%$ ee [chiral stationary phase HPLC (Daicel AS)]; $R_{f}=0.32\left(n\right.$-pentane-EtOAc, 3:1); $[\alpha]_{\mathrm{D}}{ }^{22}$ $+118.0\left(c 0.75, \mathrm{CHCl}_{3}\right)$. 
IR (ATR): 3363, 2898, 1702, 1596, 1494, 1447, 1373, 1304, 1245, $1175,1116,1034,981,927,869,785,749,693 \mathrm{~cm}^{-1}$.

${ }^{1} \mathrm{H}$ NMR $\left(600 \mathrm{MHz}, \mathrm{CDCl}_{3}\right): \delta=1.26\left(\mathrm{t}, J=7.2 \mathrm{~Hz}, 3 \mathrm{H}, \mathrm{CH}_{3}\right)$, $2.91(\mathrm{t}, J=8.4 \mathrm{~Hz}, 1 \mathrm{H}, \mathrm{NCHCH}), 3.22($ br s, $1 \mathrm{H}, \mathrm{OH}), 4.15(\mathrm{q}$ $\left.J=7.2 \mathrm{~Hz}, 2 \mathrm{H}, \mathrm{CH}_{2}\right), 5.16(\mathrm{~d}, J=8.4 \mathrm{~Hz}, 1 \mathrm{H}, \mathrm{NCHPh}), 5.41(\mathrm{~d}$, $J=16.2 \mathrm{~Hz}, 1 \mathrm{H}, \mathrm{CHCOOEt}), 5.89\left(\mathrm{~s}, 2 \mathrm{H}, \mathrm{CH}_{2}\right), 6.62(\mathrm{~s}, 1 \mathrm{H}$, $\left.\mathrm{CH}_{\mathrm{Ar}}\right), 6.65\left(\mathrm{~s}, 2 \mathrm{H}, \mathrm{CH}_{\mathrm{Ar}}\right), 7.13\left(\mathrm{t}, J=7.8 \mathrm{~Hz}, 1 \mathrm{H}, \mathrm{CH}_{\mathrm{Ar}}\right), 7.19(\mathrm{dd}$, $J=9.0,16.2 \mathrm{~Hz}, 1 \mathrm{H}, \mathrm{CH}=\mathrm{CHCOOEt}), 7.26-7.47\left(\mathrm{~m}, 9 \mathrm{H}, \mathrm{CH}_{\mathrm{Ar}}\right)$.

${ }^{13} \mathrm{C} \mathrm{NMR}\left(150 \mathrm{MHz}, \mathrm{CDCl}_{3}\right): \delta=14.2\left(\mathrm{CH}_{3}\right), 59.7(\mathrm{NCHCH}), 60.5$ $\left(\mathrm{CH}_{2}\right), 65.0(\mathrm{NCHPh}), 80.8(\mathrm{COH}), 101.2\left(\mathrm{CH}_{2}\right), 106.7\left(\mathrm{CH}_{\mathrm{Ar}}\right)$, $108.3\left(\mathrm{CH}_{\mathrm{Ar}}\right), 121.4\left(\mathrm{CH}_{\mathrm{Ar}}\right), 123.9\left(2 \mathrm{C}, \mathrm{CH}_{\mathrm{Ar}}\right), 125.8\left(2 \mathrm{C}, \mathrm{CH}_{\mathrm{Ar}}\right)$, 126.0 $\left(\mathrm{CH}_{\mathrm{Ar}}\right), 126.5(\mathrm{CHCOOEt}), 128.2\left(\mathrm{CH}_{\mathrm{Ar}}\right), 128.5\left(2 \mathrm{C}, \mathrm{CH}_{\mathrm{Ar}}\right)$, $128.7\left(2 \mathrm{C}, \mathrm{CH}_{\mathrm{Ar}}\right), 130.9(\mathrm{C}), 136.9(\mathrm{C}), 139.6$ (CHCOOEt), 140.5 (C), 147.5 (C), 148.2 (C), 165.5 (COOEt), 173.7 (NCO).

MS (EI, $70 \mathrm{eV}): m / z(\%)=471(25), 454$ (19), 453 (59), 380 (18), 379 (27), 246 (28), 226 (100), 225 (92), 224 (55), 201 (38), 172 (47), 144 (27), 135 (81), 115 (35), 105 (96), 77 (44).

HRMS: $m / z[\mathrm{M}+\mathrm{H}]^{+}$calcd for $\mathrm{C}_{28} \mathrm{H}_{26} \mathrm{NO}_{6}$ : 422.1755; found: 472.1757 .

\section{Ethyl (E)-3-[(2S,3R,4R)-2-(Furan-2-yl)-4-hydroxy-5-oxo-1,4-} diphenylpyrrolidin-3-yl]acrylate $(6 \mathrm{~h})$

Following the general procedure with purification by flash chromatography (n-pentane-EtOAc, 3:1) gave $\mathbf{6 h}$ as a colorless solid; yield: $70 \mathrm{mg}(51 \%) ; \mathrm{mp} 62-65^{\circ} \mathrm{C} ; 60 \%$ ee [chiral stationary phase HPLC (Daicel AS)]; $R_{f}=0.35$ (n-pentane-EtOAc, 3:1); $[\alpha]_{\mathrm{D}}{ }^{22}$ $+32.7\left(c 0.49, \mathrm{CHCl}_{3}\right)$.

IR (ATR): 3358, 3063, 2982, 1697, 1596, 1547, 1495, 1448, 1375, $1304,1247,1175,1122,1070,1015,979,923,880,839,743,693$ $\mathrm{cm}^{-1}$.

${ }^{1} \mathrm{H}$ NMR (600 MHz, $\left.\mathrm{CDCl}_{3}\right): \delta=1.24\left(\mathrm{t}, J=7.2 \mathrm{~Hz}, 3 \mathrm{H}, \mathrm{CH}_{3}\right)$, $3.39(\mathrm{t}, J=8.4 \mathrm{~Hz}, 1 \mathrm{H}, \mathrm{NCHCH}), 3.54(\mathrm{br} \mathrm{s}, 1 \mathrm{H}, \mathrm{OH}), 4.14(\mathrm{q}$ $\left.J=7.2 \mathrm{~Hz}, 2 \mathrm{H}, \mathrm{CH}_{2}\right), 5.21(\mathrm{~d}, J=8.4 \mathrm{~Hz}, 1 \mathrm{H}, \mathrm{NCHPh}), 5.55(\mathrm{~d}$, $J=16.2 \mathrm{~Hz}, 1 \mathrm{H}, \mathrm{CHCOOEt}), 6.19-6.22\left(\mathrm{~m}, 2 \mathrm{H}, \mathrm{CH}_{\mathrm{Ar}}\right), 7.13(\mathrm{dd}$, $J=8.4,16.2 \mathrm{~Hz}, 1 \mathrm{H}, \mathrm{CH}=\mathrm{CHCOOEt}), 7.18-7.21\left(\mathrm{~m}, 3 \mathrm{H}, \mathrm{CH}_{\mathrm{Ar}}\right)$, 7.29-7.47 (m, $\left.8 \mathrm{H}, \mathrm{CH}_{\mathrm{Ar}}\right)$.

${ }^{13} \mathrm{C} \mathrm{NMR}\left(150 \mathrm{MHz}, \mathrm{CDCl}_{3}\right): \delta=14.1\left(\mathrm{CH}_{3}\right), 54.8(\mathrm{NCHCH}), 59.4$ $(\mathrm{NCHPh}), 60.5\left(\mathrm{CH}_{2}\right), 80.3(\mathrm{COH}), 110.4\left(\mathrm{CH}_{\mathrm{Ar}}\right), 111.4\left(\mathrm{CH}_{\mathrm{Ar}}\right)$, $124.9\left(2 \mathrm{C}, \mathrm{CH}_{\mathrm{Ar}}\right), 125.9\left(2 \mathrm{C}, \mathrm{CH}_{\mathrm{Ar}}\right), 126.1$ (CHCOOEt), 126.9 $\left(\mathrm{CH}_{\mathrm{Ar}}\right), 128.2\left(\mathrm{CH}_{\mathrm{Ar}}\right), 128.4\left(2 \mathrm{C}, \mathrm{CH}_{\mathrm{Ar}}\right), 128.8\left(2 \mathrm{C}, \mathrm{CH}_{\mathrm{Ar}}\right), 136.7$ (C), $139.8(\mathrm{CH}=\mathrm{CHCOOEt}), 140.3(\mathrm{C}), 143.2\left(\mathrm{CH}_{\mathrm{Ar}}\right), 148.6(\mathrm{C})$, 165.5 (COOEt), 173.3 (NCO).

MS (EI, $70 \mathrm{eV}): m / z(\%)=419$ (18), 418 (32), 417 (23), 401 (27), 400 (100), 399 (85), 354 (12), 353 (10), 324 (17), 301 (22), 299 (13), 193 (23), 192 (51), 191 (33), 173 (25), $172(31), 171(21), 170$ (11).

HRMS: $m / z[\mathrm{M}+\mathrm{H}]^{+}$calcd for $\mathrm{C}_{25} \mathrm{H}_{24} \mathrm{NO}_{5}$ : 418.1649; found: 418.1650.

tert-Butyl 3-\{(2S,3R,4R)-3-[(E)-3-Ethoxy-3-oxoprop-1-enyl]-4hydroxy-5-oxo-1,4-diphenylpyrrolidin-2-yl $\}-1 H$-indole-1-carboxylate (6i)

Following the general procedure with purification by flash chromatography ( $n$-pentane-EtOAc, 3:1) gave $6 \mathbf{i}$ as a colorless solid; yield: $110 \mathrm{mg}(59 \%) ; \mathrm{mp} 110-115^{\circ} \mathrm{C} ; 81 \%$ ee $(88 \%)$ [chiral stationary phase HPLC (Daicel AS)]; $R_{f}=0.4(n$-pentane-EtOAc, $3: 1) ;[\alpha]_{\mathrm{D}}{ }^{22}$ $+68.2\left(c 0.51, \mathrm{CHCl}_{3}\right)$.

IR (ATR): 3379, 2980, 1710, 1602, 1456, 1364, 1253, 1154, 1084, $1032,981,849,749,696 \mathrm{~cm}^{-1}$.

${ }^{1} \mathrm{H}$ NMR $\left(600 \mathrm{MHz}, \mathrm{C}_{6} \mathrm{D}_{6}\right): \delta=1.23\left(\mathrm{t}, J=7.2 \mathrm{~Hz}, 3 \mathrm{H}, \mathrm{CH}_{3}\right), 1.64$ (s, $\left.9 \mathrm{H}, \mathrm{CH}_{3}\right), 3.78(\mathrm{t}, J=8.4 \mathrm{~Hz}, 1 \mathrm{H}, \mathrm{NCHCH}), 4.13$ (br s, $1 \mathrm{H}$, $\mathrm{OH}), 4.13\left(\mathrm{q}, J=7.2 \mathrm{~Hz}, 2 \mathrm{H}, \mathrm{CH}_{2}\right), 5.40(\mathrm{~d}, J=15.6 \mathrm{~Hz}, 1 \mathrm{H}, \mathrm{CH}-$ COOEt), $5.43(\mathrm{~d}, J=8.4 \mathrm{~Hz}, 1 \mathrm{H}, \mathrm{NCHPh}), 7.10\left(\mathrm{t}, 1 \mathrm{H}, \mathrm{CH}_{\mathrm{Ar}}\right.$ ), 7.18-7.27 (m, $\left.5 \mathrm{H}, \mathrm{CH}_{\mathrm{Ar}}\right), 7.38-7.42\left(\mathrm{~m}, 5 \mathrm{H}, \mathrm{CH}_{\mathrm{Ar}}\right), 7.47$ (br s, 1 $\left.\mathrm{H}, \mathrm{CH}_{\mathrm{Ar}}\right), 7.51\left(\mathrm{~d}, J=7.2 \mathrm{~Hz}, 2 \mathrm{H}, \mathrm{CH}_{\mathrm{Ar}}\right), 7.57(\mathrm{~d}, J=7.8 \mathrm{~Hz}, 1 \mathrm{H}$, $\left.\mathrm{CH}_{\mathrm{Ar}}\right), 8.02\left(\right.$ br s, $1 \mathrm{H}, \mathrm{CH}_{\mathrm{Ar}}$ ).
${ }^{13} \mathrm{C}$ NMR (150 MHz, DMSO- $\left.d_{6}\right): \delta=14.4\left(\mathrm{CH}_{3}\right), 28.0$ [3 C, $\left.\mathrm{C}\left(\mathrm{CH}_{3}\right)_{3}\right], 56.2(\mathrm{NCHCH}), 57.4(\mathrm{NCHPh}), 60.4\left(\mathrm{CH}_{2}\right), 80.5(\mathrm{COH})$, $84.7\left[C\left(\mathrm{CH}_{3}\right)_{3}\right], 115.6\left(\mathrm{CH}_{\mathrm{Ar}}\right), 116.7(\mathrm{C}), 120.0\left(\mathrm{CH}_{\mathrm{Ar}}\right), 123.4$ $\left(\mathrm{CH}_{\mathrm{Ar}}\right), 124.2\left(2 \mathrm{C}, \mathrm{CH}_{\mathrm{Ar}}\right), 124.9\left(\mathrm{CH}_{\mathrm{Ar}}\right), 125.5(\mathrm{CHCOOEt}), 125.9$ $\left(\mathrm{CH}_{\mathrm{Ar}}\right), 126.1\left(\mathrm{CH}_{\mathrm{Ar}}\right), 126.7\left(2 \mathrm{C}, \mathrm{CH}_{\mathrm{Ar}}\right), 126.9(\mathrm{C}), 127.9\left(\mathrm{CH}_{\mathrm{Ar}}\right)$, $128.4\left(2 \mathrm{C}, \mathrm{CH}_{\mathrm{Ar}}\right), 128.6\left(2 \mathrm{C}, \mathrm{CH}_{\mathrm{Ar}}\right), 135.4(\mathrm{C}), 137.8(\mathrm{C}), 141.6$ (C), $142.2(\mathrm{CH}=\mathrm{CHCOOEt}), 149.2(C \mathrm{CO} t-\mathrm{Bu}), 165.2(\mathrm{COOEt})$, 172.9 (NCO).

MS (EI, $70 \mathrm{eV}): m / z(\%)=566(43), 466(29), 449$ (23), 448 (100), 249 (21), 220 (16).

HRMS: $m / z[\mathrm{M}+\mathrm{H}]^{+}$calcd for $\mathrm{C}_{34} \mathrm{H}_{35} \mathrm{~N}_{2} \mathrm{O}_{6}: 567.2489$; found: 567.2488 .

\section{Ethyl (E)-3-[(2S,3R,4R)-4-Hydroxy-1-(2-iodophenyl)-5-oxo-} 2,4-diphenylpyrrolidin-3-yl]acrylate (6j)

Following the general procedure with purification by flash chromatography ( $n$-pentane-EtOAc, $3: 1$ ) gave $\mathbf{6 j}$ as a colorless solid; yield: $80 \mathrm{mg}(44 \%)$; mp $92-95^{\circ} \mathrm{C} ; 88 \%$ ee $(95 \%)$ [chiral stationary phase HPLC (Daicel AS) $; ; R_{f}=0.28(n$-pentane-EtOAc, $3: 1) ;[\alpha]_{\mathrm{D}}^{22}+4.0$ (c 1.0, $\mathrm{CHCl}_{3}$ ).

IR (ATR): 3366, 2980, 2176, 2113, 1707, 1581, 1470, 1373, 1306, $1252,1174,1119,1029,980,913,869,803,748,699 \mathrm{~cm}^{-1}$.

${ }^{1} \mathrm{H}$ NMR (600 MHz, DMSO- $\left.d_{6}\right): \delta=1.11\left(\mathrm{t}, J=7.2 \mathrm{~Hz}, 3 \mathrm{H}, \mathrm{CH}_{3}\right)$, $3.19(\mathrm{t}, J=8.4 \mathrm{~Hz}, 1 \mathrm{H}, \mathrm{NCHCH}), 4.00\left(\mathrm{q}, J=7.2 \mathrm{~Hz}, 2 \mathrm{H}, \mathrm{CH}_{2}\right)$, 5.39 (d, $J=15.6 \mathrm{~Hz}, 1 \mathrm{H}, \mathrm{CHCOOEt}), 5.54(\mathrm{~d}, J=9.6 \mathrm{~Hz}, 1 \mathrm{H}$, $\mathrm{NCHPh}), 6.91\left(\mathrm{~m}, 2 \mathrm{H}, \mathrm{CH}_{\mathrm{Ar}}\right), 7.00(\mathrm{dd}, J=9.0,15.6 \mathrm{~Hz}, 1 \mathrm{H}$, $\mathrm{CH}=\mathrm{CHCOOEt}), 7.16\left(\mathrm{~m}, 1 \mathrm{H}, \mathrm{CH}_{\mathrm{Ar}}\right), 7.22\left(\mathrm{~m}, 3 \mathrm{H}, \mathrm{CH}_{\mathrm{Ar}}\right), 7.32(\mathrm{~m}$, $\left.2 \mathrm{H}, \mathrm{CH}_{\mathrm{Ar}}\right), 7.41\left(\mathrm{~m}, 2 \mathrm{H}, \mathrm{CH}_{\mathrm{Ar}}\right), 7.47\left(\mathrm{~m}, 2 \mathrm{H}, \mathrm{CH}_{\mathrm{Ar}}\right), 7.58(\mathrm{~m}, 2 \mathrm{H}$, $\left.\mathrm{CH}_{\mathrm{Ar}}\right), 7.77\left(\mathrm{~d}, J=7.8 \mathrm{~Hz}, 1 \mathrm{H}, \mathrm{CH}_{\mathrm{Ar}}\right)$.

${ }^{13} \mathrm{C} \mathrm{NMR}\left(150 \mathrm{MHz}, \mathrm{DMSO}-d_{6}\right): \delta=14.4\left(\mathrm{CH}_{3}\right), 59.5(\mathrm{NCHCH})$, $60.5\left(\mathrm{CH}_{2}\right), 65.3(\mathrm{NCHPh}), 80.1(\mathrm{COH}), 100.1(\mathrm{C}), 125.4(\mathrm{CH}-$ COOEt), $126.7\left(2 \mathrm{C}, \mathrm{CH}_{\mathrm{Ar}}\right), 126.9\left(\mathrm{CH}_{\mathrm{Ar}}\right), 127.9\left(\mathrm{CH}_{\mathrm{Ar}}\right), 128.3(2$ $\left.\mathrm{C}, \mathrm{CH}_{\mathrm{Ar}}\right), 128.7\left(2 \mathrm{C}, \mathrm{CH}_{\mathrm{Ar}}\right), 128.8\left(\mathrm{CH}_{\mathrm{Ar}}\right), 128.9\left(2 \mathrm{C}, \mathrm{CH}_{\mathrm{Ar}}\right), 129.1$ $\left(\mathrm{CH}_{\mathrm{Ar}}\right), 129.2\left(\mathrm{CH}_{\mathrm{Ar}}\right), 137.6(\mathrm{C}), 139.8\left(\mathrm{CH}_{\mathrm{Ar}}\right), 140.8(\mathrm{C}), 141.7$ $(\mathrm{CH}=\mathrm{CHCOOEt}), 141.9(\mathrm{C}), 165.2(\mathrm{COOEt}), 171.8(\mathrm{NCO})$.

MS (EI, $70 \mathrm{eV}): m / z(\%)=553(6), 536(13), 427$ (26), $426(92), 424$ (17), 308 (30), 224 (33), 203 (19), 202 (30), 180 (17), 157 (27), 144 (10), 129 (28), 128 (16), 105 (100), 77 (31).

Anal. Calcd for $\mathrm{C}_{27} \mathrm{H}_{24} \mathrm{INO}_{4}$ : C, 58.60; H, 4.37; N, 2.53. Found: C, 58.59; H, 4.09; N, 2.37.

\section{Ethyl (E)-3-[(2S,3R,4R)-4-Hydroxy-1-(4-methoxyphenyl)-5-} oxo-2,4-diphenylpyrrolidin-3-yl] acrylate (6k)

Following the general procedure with purification by flash chromatography (n-pentane-EtOAc, $3: 1$ ) gave $\mathbf{6 k}$ as a colorless solid; yield: $86 \mathrm{mg}(60 \%)$; $\mathrm{mp} 77-79{ }^{\circ} \mathrm{C} ; 81 \%$ ee $(89 \%)$ [chiral stationary phase HPLC (Daicel AS)]; $R_{f}=0.21$ (n-pentane-EtOAc, 3:1); $[\alpha]_{\mathrm{D}}^{22}+102.0\left(c 0.5, \mathrm{CHCl}_{3}\right)$.

IR (ATR): 3356, 2974, 1695, 1510, 1453, 1375, 1293, 1243, 1173, $1114,1030,981,832,795,751,697 \mathrm{~cm}^{-1}$.

${ }^{1} \mathrm{H}$ NMR (600 MHz, $\left.\mathrm{CDCl}_{3}\right): \delta=1.24\left(\mathrm{t}, J=7.2 \mathrm{~Hz}, 3 \mathrm{H}, \mathrm{CH}_{3}\right)$, $2.95(\mathrm{t}, J=8.4 \mathrm{~Hz}, 1 \mathrm{H}, \mathrm{NCHCH}), 3.34(\mathrm{br} \mathrm{s}, 1 \mathrm{H}, \mathrm{OH}), 3.72(\mathrm{~s}, 3$ $\left.\mathrm{H}, \mathrm{OCH}_{3}\right), 4.14\left(\mathrm{q}, J=7.2 \mathrm{~Hz}, 2 \mathrm{H}, \mathrm{CH}_{2}\right), 5.18(\mathrm{~d}, J=8.4 \mathrm{~Hz}, 1 \mathrm{H}$, NCHPh), $5.37(\mathrm{~d}, J=15.6 \mathrm{~Hz}, 1 \mathrm{H}, \mathrm{CHCOOEt}), 6.76(\mathrm{~d}, J=9.0 \mathrm{~Hz}$, $\left.2 \mathrm{H}, \mathrm{CH}_{\mathrm{Ar}}\right), 7.16-7.40\left(\mathrm{~m}, 11 \mathrm{H}, \mathrm{CH}_{\mathrm{Ar}}, \mathrm{CH}=\mathrm{CHCOOEt}\right), 7.47$ (d, $\left.J=7.8 \mathrm{~Hz}, 2 \mathrm{H}, \mathrm{CH}_{\mathrm{Ar}}\right)$.

${ }^{13} \mathrm{C}$ NMR $\left(150 \mathrm{MHz}, \mathrm{CDCl}_{3}\right): \delta=14.1\left(\mathrm{CH}_{3}\right), 55.3\left(\mathrm{OCH}_{3}\right), 59.6$ $(\mathrm{NCHCH}), 60.5\left(\mathrm{CH}_{2}\right), 65.6(\mathrm{NCHPh}), 80.8(\mathrm{COH}), 114.0(2 \mathrm{C}$, $\left.\mathrm{CH}_{\mathrm{Ar}}\right), 125.4\left(2 \mathrm{C}, \mathrm{CH}_{\mathrm{Ar}}\right), 125.8\left(2 \mathrm{C}, \mathrm{CH}_{\mathrm{Ar}}\right), 126.3$ (CHCOOEt), $127.3\left(2 \mathrm{C}, \mathrm{CH}_{\mathrm{Ar}}\right), 128.2\left(\mathrm{CH}_{\mathrm{Ar}}\right), 128.3\left(\mathrm{CH}_{\mathrm{Ar}}\right), 128.5\left(2 \mathrm{C}, \mathrm{CH}_{\mathrm{Ar}}\right)$, $128.8\left(2 \mathrm{C}, \mathrm{CH}_{\mathrm{Ar}}\right), 129.9(\mathrm{C}), 137.2(\mathrm{C}), 139.8(\mathrm{CH}=\mathrm{CHCOOEt})$, 140.7 (C), 157.5 (C), 165.5 (COOEt), 173.8 (NCO).

MS (EI, $70 \mathrm{eV}): m / z(\%)=458(17), 457$ (100), $456(36), 340$ (12), 256 (16), 255 (16), 213 (12), 212 (36), 211 (34), 210 (14), 197 (15), $196(17)$ 
HRMS: $m / z[\mathrm{M}+\mathrm{H}]^{+}$calcd for $\mathrm{C}_{28} \mathrm{H}_{28} \mathrm{NO}_{5}$ : 458.1962; found: 458.1962 .

Ethyl (E)-3-[(2S,3R,4R)-4-(2,4-Dimethoxyphenyl)-4-hydroxy-5oxo-1,2-diphenylpyrrolidin-3-yl] acrylate (6l)

Following the general procedure with purification by flash chromatography ( $n$-pentane-EtOAc, 3:1) gave $\mathbf{6 l}$ as a colorless solid; yield: $87 \mathrm{mg}(69 \%) ; \mathrm{mp} 85-87{ }^{\circ} \mathrm{C} ; 90 \%$ ee [chiral stationary phase HPLC (Daicel AS) $; R_{f}=0.12(n$-pentane-EtOAc, $3: 1) ;[\alpha]_{\mathrm{D}}{ }^{22}+77.6$ (c 0.54, $\mathrm{CHCl}_{3}$ ).

IR (ATR): 3355, 2972, 1706, 1603, 1497, 1373, 1273, 1208, 1159, $1033,976,913,831,749,696 \mathrm{~cm}^{-1}$.

${ }^{1} \mathrm{H}$ NMR $\left(600 \mathrm{MHz}, \mathrm{CDCl}_{3}\right): \delta=1.23\left(\mathrm{t}, J=7.2 \mathrm{~Hz}, 3 \mathrm{H}, \mathrm{CH}_{3}\right)$, $3.14($ br s, $1 \mathrm{H}, \mathrm{OH}), 3.28(\mathrm{t}, J=9.0 \mathrm{~Hz}, 1 \mathrm{H}, \mathrm{NCHCH}), 3.82(\mathrm{~s}, 3$ $\left.\mathrm{H}, \mathrm{OCH}_{3}\right), 3.98\left(\mathrm{~s}, 3 \mathrm{H}, \mathrm{OCH}_{3}\right), 4.13\left(\mathrm{q}, J=7.2 \mathrm{~Hz}, 2 \mathrm{H}, \mathrm{CH}_{2}\right), 5.22$ $(\mathrm{d}, J=8.4 \mathrm{~Hz}, 1 \mathrm{H}, \mathrm{NCHPh}), 5.39$ (d, $J=15.6 \mathrm{~Hz}, 1 \mathrm{H}, \mathrm{CHCOOEt})$, $6.51\left(\mathrm{dd}, J=2.4,9.0 \mathrm{~Hz}, 1 \mathrm{H}, \mathrm{CH}_{\mathrm{Ar}}\right), 6.54(\mathrm{~d}, J=2.4 \mathrm{~Hz}, 1 \mathrm{H}$, $\mathrm{CH}_{\mathrm{Ar}}$ ), 7.05 (t, $J=7.2 \mathrm{~Hz}, 1 \mathrm{H}, \mathrm{CH}_{\mathrm{Ar}}$ ), 7.13 (dd, $J=9.0,16.2 \mathrm{~Hz}, 1$ $\mathrm{H}, \mathrm{CH}=\mathrm{CHCOOEt}), 7.16-7.25\left(\mathrm{~m}, 7 \mathrm{H}, \mathrm{CH}_{\mathrm{Ar}}\right), 7.31(\mathrm{~d}, J=7.8 \mathrm{~Hz}$, $\left.2 \mathrm{H}, \mathrm{CH}_{\mathrm{Ar}}\right), 7.45\left(\mathrm{~d}, J=8.4 \mathrm{~Hz}, 1 \mathrm{H}, \mathrm{CH}_{\mathrm{Ar}}\right)$.

${ }^{13} \mathrm{C}$ NMR $\left(150 \mathrm{MHz}, \mathrm{CDCl}_{3}\right): \delta=14.2\left(\mathrm{CH}_{3}\right), 55.4\left(\mathrm{OCH}_{3}\right), 55.8$ $(\mathrm{NCHCH}), 55.8\left(\mathrm{OCH}_{3}\right), 60.4\left(\mathrm{CH}_{2}\right), 65.3(\mathrm{NCHPh}), 79.4(\mathrm{COH})$ $99.5\left(\mathrm{CH}_{\mathrm{Ar}}\right), 104.5\left(\mathrm{CH}_{\mathrm{Ar}}\right), 121.3(\mathrm{C}), 123.6\left(2 \mathrm{C}, \mathrm{CH}_{\mathrm{Ar}}\right), 125.3$ $\left(\mathrm{CH}_{\mathrm{Ar}}\right), 125.8(\mathrm{CHCOOEt}), 127.3\left(2 \mathrm{C}, \mathrm{CH}_{\mathrm{Ar}}\right), 127.9\left(\mathrm{CH}_{\mathrm{Ar}}\right), 128.0$ $\left(\mathrm{CH}_{\mathrm{Ar}}\right), 128.5\left(2 \mathrm{C}, \mathrm{CH}_{\mathrm{Ar}}\right), 128.7\left(2 \mathrm{C}, \mathrm{CH}_{\mathrm{Ar}}\right), 137.4(\mathrm{C}), 138.3(\mathrm{C})$ $141.1(\mathrm{CH}=\mathrm{CHCOOEt}), 156.4$ (C), $161.0(\mathrm{C}), 165.7$ (COOEt) 174.3 (NCO).

MS (EI, $70 \mathrm{eV}): m / z(\%)=469$ (16), 468 (12), $286(21), 285$ (20), 284 (13), 166 (100), 165 (70), 164 (77).

Anal. Calcd for $\mathrm{C}_{29} \mathrm{H}_{29} \mathrm{NO}_{6}$ : C, 71.44; $\mathrm{H}, 6.00 ; \mathrm{N}, 2.87$. Found: C, 70.94; H, 6.11; N, 2.73.

\section{Ethyl $(E)-3-[(2 S, 3 R, 4 R)-4-H y d r o x y-5-0 x 0-1,2-d i p h e n y l-4-(2-$} tolyl)pyrrolidin-3-yl]acrylate $(6 \mathrm{~m})$

Following the general procedure with purification by flash chromatography (n-pentane-EtOAc, $3: 1)$ gave $\mathbf{6 m}$ as a colorless solid; yield: $107 \mathrm{mg}(73 \%) ; \mathrm{mp} 72-75{ }^{\circ} \mathrm{C} ; 93 \%$ ee [chiral stationary phase HPLC (Daicel AS)]; $R_{f}=0.38$ (n-pentane-EtOAc, 3:1); $[\alpha]_{\mathrm{D}}{ }^{22}$ $+109.6\left(c 0.5, \mathrm{CHCl}_{3}\right)$.

IR (ATR): 3363, 2916, 1697, 1597, 1495, 1454, 1373, 1277, 1249 , $1219,1170,1120,1084,1032,981,905,870,766,694 \mathrm{~cm}^{-1}$.

${ }^{1} \mathrm{H}$ NMR (600 MHz, $\left.\mathrm{CDCl}_{3}\right): \delta=1.24\left(\mathrm{t}, J=7.2 \mathrm{~Hz}, 3 \mathrm{H}, \mathrm{CH}_{3}\right)$, $2.37\left(\mathrm{~s}, 3 \mathrm{H}, \mathrm{CH}_{3}\right), 2.95(\mathrm{t}, J=8.4 \mathrm{~Hz}, 1 \mathrm{H}, \mathrm{NCHCH}), 3.49$ (br s, 1 $\mathrm{H}, \mathrm{OH}), 4.13\left(\mathrm{~m}, 2 \mathrm{H}, \mathrm{CH}_{2}\right), 5.23(\mathrm{~d}, J=8.4 \mathrm{~Hz}, 1 \mathrm{H}, \mathrm{NCHPh}), 5.37$ (d, $J=16.2 \mathrm{~Hz}, 1 \mathrm{H}, \mathrm{CHCOOEt}), 7.07-7.33\left(\mathrm{~m}, 15 \mathrm{H}, \mathrm{CH}_{\mathrm{Ar}}\right.$, $\mathrm{CH}=\mathrm{CHCOOEt}$ ).

${ }^{13} \mathrm{C}$ NMR $\left(150 \mathrm{MHz}, \mathrm{CDCl}_{3}\right): \delta=14.1\left(\mathrm{CH}_{2} \mathrm{CH}_{3}\right), 21.6\left(\mathrm{ArCH}_{3}\right)$, $59.7(\mathrm{NCHCH}), 60.5\left(\mathrm{CH}_{2}\right), 65.1(\mathrm{NCHPh}), 80.8(\mathrm{COH}), 122.9$ $\left(\mathrm{CH}_{\mathrm{Ar}}\right), 123.8\left(2 \mathrm{C}, \mathrm{CH}_{\mathrm{Ar}}\right), 125.9\left(\mathrm{CH}_{\mathrm{Ar}}\right), 126.3(\mathrm{CHCOOEt}), 126.4$ $\left(\mathrm{CH}_{\mathrm{Ar}}\right), 127.1\left(2 \mathrm{C}, \mathrm{CH}_{\mathrm{Ar}}\right), 128.2\left(\mathrm{CH}_{\mathrm{Ar}}\right), 128.3\left(\mathrm{CH}_{\mathrm{Ar}}\right), 128.6(2 \mathrm{C}$, $\left.\mathrm{CH}_{\mathrm{Ar}}\right), 128.8\left(2 \mathrm{C}, \mathrm{CH}_{\mathrm{Ar}}\right), 129.0\left(\mathrm{CH}_{\mathrm{Ar}}\right), 137.0(\mathrm{C}), 137.3(\mathrm{C}), 138.0$ (C), $139.8(\mathrm{CH}=\mathrm{CHCOOEt}), 140.5$ (C), 165.5 (COOEt), 173.9 (NCO).

MS (EI, $70 \mathrm{eV}): m / z(\%)=441$ (27), 423 (24), 203 (19), 202 (49), 182 (49), 157 (23), 129 (23), 119 (100), 91 (15).

HRMS: $m / z[\mathrm{M}+\mathrm{Na}]^{+}$calcd for $\mathrm{C}_{28} \mathrm{H}_{27} \mathrm{NO}_{4} \mathrm{Na}$ : 464.1832; found: 464.1832 .

\section{Ethyl $(E)-3-[(2 S, 3 R, 4 R)-4-H y d r o x y-5-0 x 0-2,4-d i p h e n y l-1-t o-$} sylpyrrolidin-3-yl]acrylate (6n)

Following the general procedure with purification by flash chromatography (n-pentane-EtOAc, 3:1) gave $\mathbf{6 n}$ as a colorless solid; yield: $55 \mathrm{mg}(33 \%) ; \mathrm{mp} 72-75{ }^{\circ} \mathrm{C} ; 96 \%$ [chiral stationary phase HPLC (Daicel AS)], $R_{f}=0.36\left(n\right.$-pentane-EtOAc, 3:1); $[\alpha]_{\mathrm{D}}^{22}$ $+14.0\left(c 0.5, \mathrm{CHCl}_{3}\right)$.
IR (ATR): 3413, 2982, 2307, 2173, 1716, 1656, 1597, 1492, 1453 , $1365,1308,1170,1087,1034,980,868,803,751,700,662 \mathrm{~cm}^{-1}$.

${ }^{1} \mathrm{H} \mathrm{NMR}\left(600 \mathrm{MHz}, \mathrm{CDCl}_{3}\right): \delta=1.23\left(\mathrm{t}, J=7.2 \mathrm{~Hz}, 3 \mathrm{H}, \mathrm{CH}_{2} \mathrm{CH}_{3}\right)$, $2.44\left(\mathrm{~s}, 3 \mathrm{H}, \mathrm{ArCH}_{3}\right), 2.91(\mathrm{t}, J=8.4 \mathrm{~Hz}, 1 \mathrm{H}, \mathrm{NCHCH}$ ), 3.00 (br s, $1 \mathrm{H}, \mathrm{OH}), 4.12\left(\mathrm{~m}, 2 \mathrm{H}, \mathrm{CH}_{2}\right), 5.20(\mathrm{~d}, J=8.4 \mathrm{~Hz}, 1 \mathrm{H}, \mathrm{NCHPh})$, $5.33(\mathrm{~d}, J=16.2 \mathrm{~Hz}, 1 \mathrm{H}, \mathrm{CHCOOEt}), 7.01$ (dd, $J=9,15.6 \mathrm{~Hz}, 1$ $\mathrm{H}, \mathrm{CH}=\mathrm{CHCOOEt}), 7.24-7.35\left(\mathrm{~m}, 12 \mathrm{H}, \mathrm{CH}_{\mathrm{Ar}}\right), 7.73(\mathrm{~d}, J=8.4 \mathrm{~Hz}$, $2 \mathrm{H}, \mathrm{CH}_{\mathrm{Ar}}$ ).

${ }^{13} \mathrm{C}$ NMR $\left(150 \mathrm{MHz}, \mathrm{CDCl}_{3}\right): \delta=14.1\left(\mathrm{CH}_{2} \mathrm{CH}_{3}\right), 21.7\left(\mathrm{ArCH}_{3}\right)$, $58.9(\mathrm{NCHCH}), 60.6\left(\mathrm{CH}_{2}\right), 65.1(\mathrm{NCHPh}), 80.6(\mathrm{COH}), 125.7(2$ $\left.\mathrm{C}, \mathrm{CH}_{\mathrm{Ar}}\right), 126.8\left(2 \mathrm{C}, \mathrm{CH}_{\mathrm{Ar}}\right), 127.1(\mathrm{CHCOOEt}), 128.5\left(\mathrm{CH}_{\mathrm{Ar}}\right)$, $128.6\left(2 \mathrm{C}, \mathrm{CH}_{\mathrm{Ar}}\right), 128.6\left(2 \mathrm{C}, \mathrm{CH}_{\mathrm{Ar}}\right), 128.7\left(\mathrm{CH}_{\mathrm{Ar}}\right), 128.8(2 \mathrm{C}$, $\left.\mathrm{CH}_{\mathrm{Ar}}\right), 129.4\left(2 \mathrm{C}, \mathrm{CH}_{\mathrm{Ar}}\right), 134.7(\mathrm{C}), 137.9(\mathrm{CH}=\mathrm{CHCOOEt}), 138.0$ (C), 138.0 (C), 145.4 (C), 165.2 (COOEt), 173.1 (NCO).

MS (ESI): $m / z=544\left([\mathrm{M}+\mathrm{K}]^{+}\right), 506\left([\mathrm{M}+\mathrm{H}]^{+}\right)$.

HRMS: $m / z[\mathrm{M}+\mathrm{H}]^{+}$calcd for $\mathrm{C}_{28} \mathrm{H}_{28} \mathrm{NO}_{6} \mathrm{~S}$ : 506.1632 ; found: 506.1625 .

\section{Acknowledgment}

We thank the former Degussa AG and BASF SE for the donation of the chemicals. D.E. thanks the European Research Council for an ERC Advanced Grant (DOMINOCAT).

Supporting Information for this article is available online at http://www.thieme-connect.com/ejournals/toc/synthesis.

\section{References}

(1) (a) Omura, S.; Fujimoto, T.; Otoguro, K.; Matsuzaki, K.; Moriguchi, R.; Tanaka, H.; Sasaki, Y. J. Antibiot. 1991, 44, 113. (b) Corey, E. J.; Reichard, G. A. J. Am. Chem. Soc. 1992, 114, 10677.

(2) (a) Yang, M.-H.; Chen, Y.-Y.; Huang, L. Phytochemistry 1988, 24, 445. (b) Hartwig, W.; Born, L. J. Org. Chem. 1987, $52,4352$.

(3) (a) Schneider, H. H.; Schmiechen, R.; Brezinski, M.; Seidler, J. Eur. J. Pharmacol. 1986, 127, 105. (b) Mulzer, J.; Zuhse, R.; Schmiechen, R. Angew. Chem., Int. Ed. Engl. 1992, 31, 870. (c) Meyers, A. I.; Snyder, L. J. Org. Chem. 1993, $58,36$.

(4) Dwoskin, L. P.; Teng, L.; Buxton, S. T.; Crooks, P. A. J. Pharmacol. Exp. Ther. 1999, 288, 905.

(5) Reichard, G. A.; Paliwal, S.; Shih, N.-Y.; Xiao, D.; Tsui, H.C.; Shah, S.; Wang, C.; Wrobleski, M. L. WO 2003,042,173, 2003.

(6) Winn, M.; Boyd, S. A.; Hutchins, C. W.; Hwan-Soo, J.; Tasker, A. S.; Von Geldern, T. W.; Kester, J.; Sorensen, B. K.; Szszepankiewicz, B. G.; Henry, K. J.; Lui, G.; Wittenberger, S. J.; King, S. A.; Janus, T. J.; Padley, R. J. WO 2002,017,912, 2002.

(7) Via cyclization: (a) Hashmi, A. S. K.; Yang, W.; Yu, Y.; Hansmann, M. M.; Rudolph, M.; Rominger, F. Angew. Chem. Int. Ed. 2013, 52, 1329. Via cycloaddition: (b) Roberson, C.; Woerpel, K. A. J. Org. Chem. 1999, 64, 1434. (c) Candeias, N. R.; Gois, P. M. P.; Afonso, C. A. M. J. Org. Chem. 2006, 71, 5489. (d) Basavaiah, D.; Rao, J. S. Tetrahedron Lett. 2004, 45, 1621. Via ring expansion: (e) Rai, V. K.; Rai, P. K.; Bajaj, S.; Kumar, A. Green Chem. 2011, 13, 1217. (f) Choi, H. G.; Park, D.-S.; Lee, W. K.; Sim, T. Tetrahedron Lett. 2013, 54, 5775. Via ring contraction: (g) Brown, G. R.; Foubister, A. J.; Wright, B. J. Chem. Soc., Chem. Commun. 1984, 1373. Via multicomponent reactions: (h) Pelletier, S. M. C.; Ray, P. C.; Dixon, D. J. Org. Lett. 2009, 11, 4512. (i) Younai, A.; Chin, 
G. F.; Shaw, J. T. J. Org. Chem. 2010, 75, 8333. (j) He, M.; Rommel, M.; Bode, J. W. Heterocycles 2012, 86, 1689.

(8) For typical examples, see: (a) Anderson, J. C.; Horsfall, L. R.; Kalogirou, A. S.; Mills, M. S.; Stepney, G. J.; Tizzard, G. J. J. Org. Chem. 2012, 77, 6186. (b) Clayden, J.; Watson, D. W.; Helliwell, M.; Chambers, M. Chem. Commun. 2003, 2582.

(9) For selected examples, see: (a) Yang, L.; Wang, D.-X.; Huang, Z.-T.; Wang, M.-X. J. Am. Chem. Soc. 2009, 131, 10390. (b) Bhagwat, S. S.; Gude, C.; Chan, K. Tetrahedron Lett. 1996, 37, 4627.

(10) For selected general reviews, see: (a) Berkessel, A.; Gröger, H. Asymmetric Organocatalysis; Wiley-VCH: Weinheim, 2005. (b) Dalko, P. I. Enantioselective Organocatalysis; Wiley-VCH: Weinheim, 2007. (c) Special issue, List, B., Ed.; Chem. Rev. 2007, 107, 5413. (d) Vicario, J. L.; Badia, D.; Carillo, L. Synthesis 2007, 2065. (e) Tsogoeva, S. B. Eur. J. Org. Chem. 2007, 1701. (f) Dondoni, A.; Massi, A. Angew. Chem. Int. Ed. 2008, 47, 4638. (g) MacMillan, D. W. C. Nature (London) 2008, 455, 304. (h) Barbas, C. F. III Angew. Chem. Int. Ed. 2008, 47, 42. (i) Enders, D.; Narine, A. A. J. Org. Chem. 2008, 73, 7857. (j) Melchiorre, P.; Marigo, M.; Carlone, A.; Bartoli, G. Angew. Chem. Int. Ed. 2008, 47, 6138. (k) Jørgensen, K. A.; Bertelsen, S. Chem. Soc. Rev. 2009, 38, 2178. (1) Bella, M.; Gasperi, T. Synthesis 2009, 1583. (m) Roca-Lopez, D.; Sadaba, D.; Delso, I.; Herrera, R. P.; Tejero, T.; Merino, P. Tetrahedron: Asymmetry 2010, 21, 2561. (n) Marcelli, T.; Hiemstra, H. Synthesis 2010, 1229. (o) Nielsen, M.; Worgull, D.; Zweifel, T.; Gschwend, B.; Bertelsen, S.; Jørgensen, K. A. Chem. Commun. 2011, 47, 632. (p) Ramachary, D. B.; Jain, S. Org. Biomol. Chem. 2011, 9, 1277. (q) Moyano, A.; Rios, R. Chem. Rev. 2011, 111, 4703. (r) Pellissier, H. Tetrahedron 2012, 68, 2197. (s) Scheffler, U.; Mahrwald, R. Chem. Eur. J. 2013, 19, 14346.

(11) For selected reviews on organocatalytic domino reactions, see: (a) Lu, L.-Q.; Chen, J.-R.; Xiao, W.-J. Acc. Chem. Res. 2012, 45, 1278. (b) Pellissier, H. Adv. Synth. Catal. 2012, 354, 237. (c) Enders, D.; Grossmann, A. Angew. Chem. Int. Ed. 2012, 51, 314. (d) Jørgensen, K. A.; Albrecht, Ł.; Jiang, H. Angew. Chem. Int. Ed. 2011, 50, 8492. (e) Grondal, C.; Jeanty, M.; Enders, D. Nature Chem. 2010, 2, 167. (f) Alba, A.-N.; Companyo, X.; Viciano, M.; Rios, R. Curr. Org. Chem. 2009, 13, 1432. (g) Yu, X.; Wang, W. Org. Biomol. Chem. 2008, 6, 2037. (h) Enders, D.; Grondal, C.; Hüttl, M. Angew. Chem. Int. Ed. 2007, 46, 1570.

(12) For examples of asymmetric secondary amine catalyzed simple domino reactions, see: (a) Alexakis, A.; Lefranc, A.; Guénée, L. Org. Lett. 2013, 15, 2172. (b) Lee, H.-J.; Cho, C.-W. Eur. J. Org. Chem. 2013, in press; doi: 10.1002/ejoc.201301260. (c) Wu, L.; Wang, Y.; Song, H.;
Tang, L.; Zhou, Z.; Tang, C. Chem. Asian J. 2013, 8, 2204. (d) Zhang, X.; Song, X.; Li, H.; Zhang, S.; Chen, X.; Yu, X.; Wang, W. Angew. Chem. Int. Ed. 2012, 51, 7282. (e) Wang, C.; Yang, X.; Raabe, G.; Enders, D. Adv. Synth. Catal. 2012, 354, 2629. (f) Rueping, M.; Kuenkel, A.; Tato, F.; Bats, J. W. Angew. Chem. Int. Ed. 2009, 48, 3699. (g) Enders, D.; Wang, C.; Raabe, G. Synthesis 2009, 4119. (h) Li, H.; Zu, L.; Xie, H.; Wang, J.; Wang, W. Chem. Commun. 2008, 5636. (i) Wang, W.; Li, H.; Wang, J.; Zu, L. J. Am. Chem. Soc. 2006, 128, 10354. (j) Bui, T.; Barbas, C. F. III Tetrahedron Lett. 2000, 41, 6951.

(13) For examples of asymmetric secondary amine catalyzed triple domino reactions, see: (a) Dong, L.-J.; Fan, T.-T.; Wang, C.; Sun, J. Org. Lett. 2013, 15, 204. (b) Enders, D.; Joie, C.; Deckers, K. Chem. Eur. J. 2013, 19, 10818. (c) Chatterjee, I.; Bastida, D.; Melchiorre, P. Adv. Synth. Catal. 2013, 355, 3124. (d) Jiang, K.; Jia, Z. J.; Chen, S.; Wu, L.; Chen, Y. C. Chem. Eur. J. 2010, 16, 2852. (e) Urushima, T.; Sakamoto, D.; Ishikawa, H.; Hayashi, Y. Org. Lett. 2010, 12, 4588. (f) Bencivenni, G.; Wu, L. Y.; Mazzanti, A.; Giannichi, B.; Pesciaioli, F.; Song, M. P.; Bartoli, G.; Melchiorre, P. Angew. Chem. Int. Ed. 2009, 48, 7200. (g) Enders, D.; Hüttl, M. R. M.; Raabe, G.; Bats, J. W. Adv. Synth. Catal. 2008, 350, 267. (h) Carlone, A.; Cabrera, S.; Marigo, M.; Jorgensen, K. A. Angew. Chem. Int. Ed. 2007, 46, 1101. (i) Enders, D.; Hüttl, M. R. M.; Grondal, C.; Raabe, G. Nature (London) 2006, 441, 861.

(14) For examples of asymmetric secondary amine catalyzed quadruple domino reactions, see: (a) Zeng, X.; Ni, Q.; Raabe, G.; Enders, D. Angew. Chem. Int. Ed. 2013, 52, 2977. (b) Erdmann, N.; Philipps, A. R.; Atodiresei, I.; Enders, D. Adv. Synth. Catal. 2013, 355, 847. (c) Enders, D.; Greb, A.; Deckers, K.; Selig, P.; Merkens, C. Chem. Eur. J. 2012, 18, 10226. (d) Rueping, M.; Haack, K.; Ieasuwan, W.; Sundén, H.; Blanco, M.; Shoepke, F. R. Chem. Commun. 2011, 47, 3828. (e) Krüll, R.; Bettray, W.; Enders, D. Synthesis 2010, 567. (f) Wang, C.; Mukanova, M.; Greb, A.; Enders, D. Chem. Commun. 2010, 46, 2477. (g) Jiang, K.; Jia, Z.-J.; Yin, X.; Wu, L.; Chen, Y. C. Org. Lett. 2010, 12, 2766. (h) Zhang, F.-L.; Xu, A.-W.; Gong, Y.-F.; Wei, M.-H.; Yang, X.-L. Chem. Eur. J. 2009, 15, 6815. (i) Kotame, P.; Hong, B.-C.; Liao, J.-H. Tetrahedron Lett. 2009, 50, 704.

(15) For reviews on organocatalytic asymmetric aza-Michael reactions, see: (a) Kwong, F. Y.; Wang, J.; Li, P.; Choy, P. Y.; Chan, A. S. C. ChemCatChem 2012, 4, 917. (b) Enders, D.; Wang, C.; Liebich, J. X. Chem. Eur. J. 2009, 15, 11058. (c) Krishna, P. R.; Sreeshailam, A.; Srinivas, R. Tetrahedron 2009, 65, 9657.

(16) Baslé, O.; Raimondi, W.; Sanchez Duque, M. M.; Bonne, D.; Constantieux, T.; Rodriguez, J. Org. Lett. 2010, 12, 5246. 